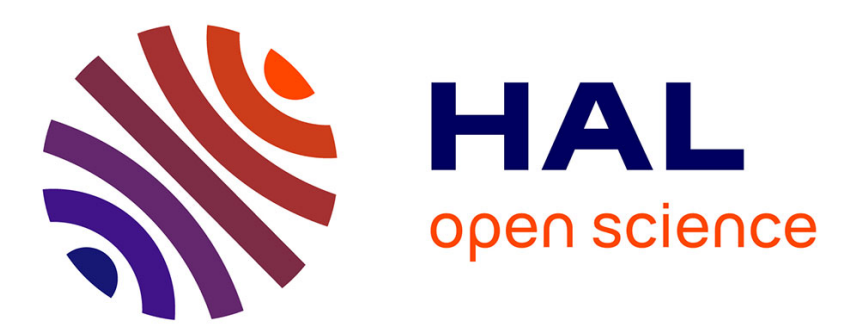

\title{
Formulation and accuracy of on-surface radiation conditions for acoustic multiple scattering problems
}

\author{
Hasan Alzubaidi, Xavier Antoine, Chokri Chniti
}

\section{To cite this version:}

Hasan Alzubaidi, Xavier Antoine, Chokri Chniti. Formulation and accuracy of on-surface radiation conditions for acoustic multiple scattering problems. Applied Mathematics and Computation, 2016, 277, pp.82-100. 10.1016/j.amc.2015.12.023 . hal-01244517

\section{HAL Id: hal-01244517 \\ https://hal.science/hal-01244517}

Submitted on 3 Feb 2016

HAL is a multi-disciplinary open access archive for the deposit and dissemination of scientific research documents, whether they are published or not. The documents may come from teaching and research institutions in France or abroad, or from public or private research centers.
L'archive ouverte pluridisciplinaire HAL, est destinée au dépôt et à la diffusion de documents scientifiques de niveau recherche, publiés ou non, émanant des établissements d'enseignement et de recherche français ou étrangers, des laboratoires publics ou privés. 


\title{
Formulation and accuracy of On-Surface Radiation Conditions for acoustic multiple scattering problems
}

\author{
Hasan Alzubaidi ${ }^{\mathrm{a}}$, Xavier Antoine ${ }^{\mathrm{b}}$, Chokri Chniti ${ }^{\mathrm{a}, \mathrm{c}}$ \\ ${ }^{a}$ Department of Mathematics, University College in Qunfudah, Umm Al-Qura University, Saudi Arabia. \\ ${ }^{b}$ Université de Lorraine, Institut Elie Cartan de Lorraine, UMR 7502, Vandoeuvre-lès-Nancy, F-54506, France. \\ ${ }^{c}$ Preparatory Institute for Engineering Studies of Nabeul, Tunisia.
}

\begin{abstract}
The aim of this paper is 1) to address an extension of the On-Surface Radiation Condition (OSRC) method to multiple scattering by a cluster of convex obstacles and 2) to analyze the numerical accuracy of the formulation for some model problems. First, the acoustic scattering problem is considered and materials about integral equations and OSRCs are recalled. Then, an OSRC integral equation formulation is developed for the Dirichlet multiple scattering problem. Finally, an extensive numerical study of the method is proposed in the special case of the scattering by circular cylinders. Even if this situation is not completely general, it provides an assessment of the accuracy of the approximation, with respect to various parameters, including thanks to the choice of the OSRC operators.
\end{abstract}

Keywords: acoustics, multiple scattering, Helmholtz equation, on-surface radiation conditions, numerical method

\section{Contents}

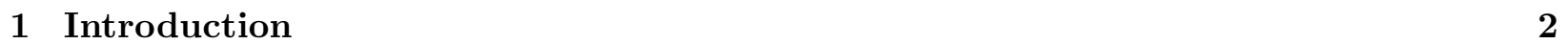

\begin{tabular}{|lll}
2 & Integral equation formulations for multiple scattering & 3
\end{tabular}

\begin{tabular}{|lll|}
\hline 3 & OSRCs for single scattering: overview and standard operators & 6
\end{tabular}

\begin{tabular}{|llll}
4 & OSRCs and multiple scattering for the Dirichlet boundary-value problem & 8
\end{tabular}

5 The example of scattering by a cluster of circular cylinders 10

5.1 Numerical approximation for a cluster of circular cylinders . . . . . . . . . . . . 10

5.2 Example 1: scattering by two circular cylinders . . . . . . . . . . . . . . . . . 11

5.3 Example 2: scattering by a triangular lattice of circular cylinders . . . . . . . . . . 12

5.4 Example 3: scattering by randomly distributed circular cylinders $\ldots \ldots$. . . . . . 12

$\begin{array}{lll}6 & \text { Conclusion } & 13\end{array}$

Email addresses: hmzubaidi@uqu.edu.sa (Hasan Alzubaidi), xavier.antoine@univ-lorraine.fr (Xavier Antoine), cachniti@uqu.edu.sa (Chokri Chniti) 


\section{Introduction}

We consider $M$ regular, bounded and disjoint single scatterers $\Omega_{p}^{-}, p=1, \ldots, M$, distributed in the two-dimensional space, with boundary $\Gamma_{p}:=\partial \Omega_{p}^{-}$. The global scatterer $\Omega^{-}$is then defined as the cluster built with the $M$ separate obstacles: $\Omega^{-}=\cup_{p=1}^{M} \Omega_{p}^{-}$. Its boundary is $\Gamma=\cup_{p=1}^{M} \Gamma_{p}$. The homogeneous isotropic unbounded domain of propagation is set to: $\Omega^{+}=\mathbb{R}^{2} \backslash \overline{\Omega^{-}}$. We assume that the scatterers are sound-soft (Dirichlet boundary condition). The extension to other situations (e.g. sound-hard scatterers, impedance boundary conditions) will be considered in a forthcoming paper. We now choose a time-harmonic incident wave $u^{\text {inc }}$ illuminating $\Omega^{-}$. The time dependence is $e^{-i \omega t}$, where $\omega$ is the wave pulsation and $k$ is the wavenumber. The sound-soft multiple scattering problem of $u^{\text {inc }}$ by $\Omega^{-}$leads to calculating the scattered wavefield $u$ as the solution to the boundary-value problem [14, 39]

$$
\left\{\begin{array}{l}
\left(\Delta+k^{2}\right) u=0, \text { in } \Omega^{+}, \\
u=-u^{\text {inc }}, \text { on } \Gamma \\
\lim _{\|\mathbf{x}\| \rightarrow+\infty}\|\mathbf{x}\|^{1 / 2}\left(\nabla u \cdot \frac{\mathbf{x}}{\|\mathbf{x}\|}-i k u\right)=0 .
\end{array}\right.
$$

The operator $\Delta=\partial_{x_{1}}^{2}+\partial_{x_{2}}^{2}$ is the Laplace operator, $\left(\Delta+k^{2}\right)$ being then the Helmholtz operator. The gradient operator is $\nabla$ and the euclidian norm is $\|\mathbf{x}\|=\sqrt{\mathbf{x} \cdot \mathbf{x}}$, where $\mathbf{x} \cdot \mathbf{y}$ designates the inner product of two vectors $\mathbf{x}$ and $\mathbf{y}$ of $\mathbb{R}^{2}$. The last equation of (1) is the well-known Sommerfeld's radiation condition at infinity. This asymptotic condition ensures the uniqueness of the scattered wave $u$ to problem (1) (see e.g. [22, 44]).

Multiple scattering is a clearly difficult physical and numerical problem since interactions between the single scatterers generate some complex scattered field structures. Consequently, the physics emerging from such global phenomenae present some specific and unusual behaviors. Various numerical techniques [1, 2, 9, 14, 15, 20, 26, 27, 30, 32, 36, 53, can be considered for predicting the multiple scattering behavior of a complex configuration. One possible approach is based on rigorous integral equation formulations. For a general boundary $\Gamma$, boundary element discretization techniques need to be used [12, 14, 22, 39, 44]. While being powerful and applicable to many situations, they also have some disadvantages. Indeed, they lead to the solution of large dense linear systems because of the nonlocal character of integral operators. This is most particularly true for problems that consider small wavelengths $(\lambda \ll 1)$, large scatterers $\left(\operatorname{size}\left(\Omega^{-}\right) \gg 1\right)$ or clusters made of many objects $(M \gg 1)$. A huge memory is then required for solving these systems, resulting in important computational times. Even if Krylov subspace solvers [10, 11, 12, 47] can be used with fast matrix-vector products algorithms (for example Multilevel Fast Multipole Methods [31] or other compression techniques [14, 30]), the resulting method is still computationally expensive. When the geometry is more trivial, simplifications can be considered in integral equation approaches. For example, explicit analytical expressions of the integral operators can be derived, and highly accurate and fast solution techniques can be developed like e.g. for disks [14, 39, 51]. Even if circular cylinders are simple geometries, the solution of such problems is still extremely useful in applications (acoustics, electromagnetics, optics, nanophotonics, elasticity) that involve many circular scatterers, modeling structured or disordered media, in particular for large values of $k$ and $M$ (see e.g. [18, 24, 25, 29, 34, 35, 36, 43, 48, 52, 54]).

The aim of this paper is to extend the method of On-Surface Radiation Conditions (OSRCs) [37] to multiple scattering by a cluster of sound-soft acoustic scatterers. Initially, the OSRC method was developed for scattering by single convex obstacles. Among the years, many improvements have 
been addressed [3, 4, 7, 13, 16, 40, 42, 46]. The main advantage of the OSRC technique is that, for single-scattering, it leads to the numerical solution of (local) partial differential operators set over the surface $\Gamma$ of the scatterer, instead of nonlocal integral operators. This is a huge difference since, indeed, the resulting complex-valued linear system that must be solved at the end is highly sparse and not dense. Hence, fast numerical solutions can be derived. The essential drawback of OSRC techniques is that the computational gain in terms of CPU time is paid by the fact that only an approximate solution to the scattering problem can be obtained, and not an exact one as for integral formulations. One of the reasons is that multiple scattering effects are non trivial to include into the OSRC methods. Some efforts have been directed to improve the accuracy of the OSRC solution when considering for example single scattering by non convex structures [5]. The aim of this paper is to rather consider another situation: scattering by a cluster of convex objects. A recent study [1] by Acosta shown that OSRCs can have a strong potential for solving multiple scattering problems. The present paper completes the contribution [1] since it provides a clearly related but also different point of view. In particular, we analyze the formulation according to the choice of the OSRC operator and the numerical study considers both small and large wavelengths as well as complex multiple scattering configurations with many scatterers... The objective of this paper is twofold. First, we want to show how to derive a generic OSRC formulation for multiple scattering problems which naturally incorporates any possible OSRC operator. Secondly, we conduct a numerical investigation of the potentiality of the proposed method for complex multiple scattering configurations with many circular cylinders and various frequency regimes. This paper is a first step towards the development of fast OSRC numerical solutions [8] involving many arbitrarily shaped scatterers that could be designed through iterative techniques (like domain decomposition methods) to benefit from the sparse structure of the OSRC matrices related to each local single scatterer. The final algorithm then would lead to only solving $M$ small size and sparse linear systems coupled through iterations instead of dense complex valued linear systems.

The structure of the paper is the following. The aim of Section 2 is to recall some results concerning integral equations for multiple scattering. In Section 3, we introduce some elements on OSRC techniques and the most standard operators that will be used. Section 4 develops the OSRC formulation for the Dirichlet multiple scattering problem. The formulation is tested in Section 5 for the scattering problem by many circular cylinders. The geometrical configurations that are considered are 1) two scatterers, 2) a triangular lattice and 3) a cluster of randomly distributed scatterers. In particular, we introduce a new OSRC operator that allows a relatively accurate representation of the scattered field for a large range of frequencies (from small to large wavenumbers $k$ ). These various tests lead to understanding the capacity of OSRC methods for solving multiple scattering problems. The paper ends by a conclusion in Section 6 .

\section{Integral equation formulations for multiple scattering}

Let us introduce $G$ as the two-dimensional free-space Green's function given by

$$
\forall \mathbf{x}, \mathbf{y} \in \mathbb{R}^{2}, \mathbf{x} \neq \mathbf{y}, \quad G(\mathbf{x}, \mathbf{y})=\frac{i}{4} H_{0}^{(1)}(k\|\mathbf{x}-\mathbf{y}\|) .
$$

The special function $H_{0}^{(1)}$ is the first-kind Hankel function of order zero. Integral equations are mainly based on the standard Helmholtz integral representation formula [22]. 
Proposition 1. If $v$ is a solution to the Helmholtz equation in an unbounded connected domain $\Omega^{+}$and if $v$ satisfies the Sommerfeld's radiation condition, then we have the following relation

$$
\int_{\Gamma}-G(\mathbf{x}, \mathbf{y}) \partial_{\mathbf{n}} v(\mathbf{y})+\partial_{\mathbf{n}_{\mathbf{y}}} G(\mathbf{x}, \mathbf{y}) v(\mathbf{y}) \mathrm{d} \Gamma(\mathbf{y})= \begin{cases}v(\mathbf{x}) & \text { if } \mathbf{x} \in \Omega^{+} \\ 0 & \text { otherwise. }\end{cases}
$$

In addition, if $v^{-}$is solution to the Helmholtz equation in a bounded domain $\Omega^{-}$, then we can write that

$$
\int_{\Gamma}-G(\mathbf{x}, \mathbf{y}) \partial_{\mathbf{n}} v^{-}(\mathbf{y})+\partial_{\mathbf{n}_{\mathbf{y}}} G(\mathbf{x}, \mathbf{y}) v^{-}(\mathbf{y}) \mathrm{d} \Gamma(\mathbf{y})= \begin{cases}0 & \text { if } \mathbf{x} \in \Omega^{+} \\ -v^{-}(\mathbf{x}) & \text { otherwise }\end{cases}
$$

The integrals on $\Gamma$ should be interpreted as duality brackets between the Sobolev spaces $H^{1 / 2}(\Gamma)$ and $H^{-1 / 2}(\Gamma)$. However, when the incident wavefield $u^{\text {inc }}$ and the curve $\Gamma$ are both regular enough, the scattered field is then smooth and the duality bracket can be identified to the (non hermitian) inner product in $L^{2}(\Gamma)$

$$
\langle f, g\rangle_{H^{-1 / 2}, H^{1 / 2}}=\int_{\Gamma} f g d \Gamma .
$$

We adopt this notation in the sequel of the paper.

Let us now introduce the volume single- and double-layer integral operators, respectively denoted by $\mathscr{L}$ and $\mathscr{M}$. They are defined by the following relations $\forall \mathbf{x} \in \mathbb{R}^{2} \backslash \Gamma$

$$
\begin{aligned}
& \mathscr{L}: \rho \quad \longmapsto \quad \mathscr{L} \rho(\mathbf{x})=\int_{\Gamma} G(\mathbf{x}, \mathbf{y}) \rho(\mathbf{y}) \mathrm{d} \Gamma(\mathbf{y}), \\
& \mathscr{M}: \lambda \longmapsto \mathscr{M} \lambda(\mathbf{x})=-\int_{\Gamma} \partial_{\mathbf{n}_{\mathbf{y}}} G(\mathbf{x}, \mathbf{y}) \lambda(\mathbf{y}) \mathrm{d} \Gamma(\mathbf{y}) .
\end{aligned}
$$

From these relations, the wavefields $v$ and $v^{-}$can be written as (see equations (2) and (3))

$$
\begin{cases}v(\mathbf{x})=-\mathscr{L}\left(\left.\partial_{\mathbf{n}} v\right|_{\Gamma}\right)(\mathbf{x})-\mathscr{M}\left(\left.v\right|_{\Gamma}\right)(\mathbf{x}), & \forall \mathbf{x} \in \Omega^{+} \\ v^{-}(\mathbf{x})=\mathscr{L}\left(\left.\partial_{\mathbf{n}} v^{-}\right|_{\Gamma}\right)(\mathbf{x})+\mathscr{M}\left(\left.v^{-}\right|_{\Gamma}\right)(\mathbf{x}), & \forall \mathbf{x} \in \Omega^{-}\end{cases}
$$

In addition, the single- and double-layer integral operators provide some outgoing solutions to the Helmholtz equation [21].

Proposition 2. For any surface densities $\rho \in H^{-1 / 2}(\Gamma)$ and $\lambda \in H^{1 / 2}(\Gamma)$, the functions $\mathscr{L} \rho$ and $\mathscr{M} \lambda$ are outgoing solutions to the Helmholtz equation in $\mathbb{R}^{2} \backslash \Gamma$.

Let us now recall the trace and normal derivative trace of the volume single- and double-layer potentials. These relations are usually called jump relations (see e.g. [21]).

Proposition 3. For any $\mathbf{x}$ in $\Gamma$, the trace and normal derivative traces of the operators $\mathscr{L}$ and $\mathscr{M}$ are such that ( \pm precise that $\mathbf{z}$ tends towards $\mathbf{x}$ from the exterior/interior of $\Gamma$ )

$$
\begin{array}{lr}
\lim _{\mathbf{z} \in \Omega^{ \pm} \rightarrow \mathbf{x}} \mathscr{L} \rho(\mathbf{z})=L \rho(\mathbf{x}), & \lim _{\mathbf{z} \in \Omega^{ \pm} \rightarrow \mathbf{x}} \mathscr{M} \lambda(\mathbf{z})=\left(\mp \frac{1}{2} I+M\right) \lambda(\mathbf{x}), \\
\lim _{\mathbf{z} \in \Omega^{ \pm} \rightarrow \mathbf{x}} \partial_{\mathbf{n}_{\mathbf{z}}} \mathscr{L} \rho(\mathbf{z})=\left(\mp \frac{1}{2} I+N\right) \rho(\mathbf{x}), & \lim _{\mathbf{z} \in \Omega^{ \pm} \rightarrow \mathbf{x}} \partial_{\mathbf{n}_{\mathbf{z}}} \mathscr{M} \lambda(\mathbf{z})=D \lambda(\mathbf{x}),
\end{array}
$$


where $I$ is given as the identity operator, for $\mathbf{x} \in \Gamma$, and

$$
\begin{array}{ll}
L \rho(\mathbf{x})=\int_{\Gamma} G(\mathbf{x}, \mathbf{y}) \rho(\mathbf{y}) \mathrm{d} \Gamma(\mathbf{y}), & M \lambda(\mathbf{x})=-\int_{\Gamma} \partial_{\mathbf{n}_{\mathbf{y}}} G(\mathbf{x}, \mathbf{y}) \lambda(\mathbf{y}) \mathrm{d} \Gamma(\mathbf{y}), \\
N \rho(\mathbf{x})=\int_{\Gamma} \partial_{\mathbf{n}_{\mathbf{x}}} G(\mathbf{x}, \mathbf{y}) \rho(\mathbf{y}) \mathrm{d} \Gamma(\mathbf{y})=-M^{*} \rho(\mathbf{x}), & D \lambda(\mathbf{x})=-\partial_{\mathbf{n}_{\mathbf{x}}} \int_{\Gamma} \partial_{\mathbf{n}_{\mathbf{y}}} G(\mathbf{x}, \mathbf{y}) \lambda(\mathbf{y}) \mathrm{d} \Gamma(\mathbf{y}) .
\end{array}
$$

Throughout the paper, the boundary integral operators are denoted by a roman letter (e.g. $L$ ) while the volume integral operators use a calligraphic letter (e.g. $\mathscr{L}$ ). The operator $M^{*}=-N$ is the adjoint operator of $M$, that is

$$
\langle g, M f\rangle_{H^{-1 / 2}, H^{1 / 2}}=\langle-N g, f\rangle_{H^{-1 / 2}, H^{1 / 2}}, \quad \forall(f, g) \in H^{1 / 2}(\Gamma) \times H^{-1 / 2}(\Gamma) .
$$

Compactness or invertibility properties of integral operators can also be obtained [14, 22, 44].

We consider here the single-layer representation of the scattered field

$$
u=\mathscr{L} \rho
$$

even if various integral equations can also be written for solving the Dirichlet problem (like the Combined Field Integral Equation (CFIE) or the Brakhage-Werner integral equation, see [11, 14, [19, 22, 33, 44, 51]). From the representation (6) and Proposition 3, one can prove that the surface density $\rho$ is equal to $\left.\left(-\partial_{\mathbf{n}} u-\partial_{\mathbf{n}} u^{\text {inc }}\right)\right|_{\Gamma}$. A first-kind integral equation, which is usually called Electric Field Integral Equation (EFIE), is based on the trace of the single-layer operator

$$
L \rho=-\left.u^{\text {inc }}\right|_{\Gamma} .
$$

The equation is well-posed and equivalent to the exterior scattering problem (1) as soon as $k$ is not an irregular interior frequency of the associated Dirichlet boundary-value problem [12, 49, When $\Omega^{-}=\bigcup_{p=1}^{M} \Omega_{p}$ is multiply connected, all the integral operators can be written by blocks. For example, the single-layer potential $\mathscr{L} \rho$ can be expressed as the sum of elementary potentials

$$
\mathscr{L} \rho=\sum_{p=1}^{M} \mathscr{L}_{p} \rho_{p},
$$

where $\rho_{p}=\left.\rho\right|_{\Gamma_{p}}$ and

$$
\mathscr{L}_{p} \rho_{p}(\mathbf{x})=\int_{\Gamma_{p}} G(\mathbf{x}, \mathbf{y}) \rho_{p}(\mathbf{y}) \mathrm{d} \mathbf{x}, \quad \forall \mathbf{x} \in \mathbb{R}^{2} \backslash \overline{\Omega_{p}} .
$$

Another way of writing equation (7) is

$$
\left(\begin{array}{cccc}
L_{1,1} & L_{1,2} & \ldots & L_{1, M} \\
L_{2,1} & L_{2,2} & \ldots & L_{2, M} \\
\vdots & \vdots & \ddots & \vdots \\
L_{M, 1} & L_{M, 2} & \ldots & L_{M, M}
\end{array}\right)\left(\begin{array}{c}
\rho_{1} \\
\rho_{2} \\
\vdots \\
\rho_{M}
\end{array}\right)=-\left(\begin{array}{c}
\left.u^{\mathrm{inc}}\right|_{\Gamma_{1}} \\
\left.u^{\mathrm{inc}}\right|_{\Gamma_{2}} \\
\vdots \\
\left.u^{\mathrm{inc}}\right|_{\Gamma_{M}}
\end{array}\right),
$$

setting $L_{p, q} \rho_{q}=\left.\left(L_{q} \rho_{q}\right)\right|_{\Gamma_{p}}$ and

$$
\forall \mathbf{x} \in \Gamma_{q}, L_{q} \rho_{q}(\mathbf{x})=\int_{\Gamma_{q}} G(\mathbf{x}, \mathbf{y}) \rho_{q}(\mathbf{x}) \mathrm{d} \mathbf{y} .
$$


Introducing some matrix operator notations, we also have the compact form of the EFIE for the acoustic multiple scattering problem

$$
L \boldsymbol{\rho}=-\mathbf{U}^{\text {inc }},
$$

where

$$
L:=\left(\begin{array}{cccc}
L_{1,1} & L_{1,2} & \ldots & L_{1, M} \\
L_{2,1} & L_{2,2} & \ldots & L_{2, M} \\
\vdots & \vdots & \ddots & \vdots \\
L_{M, 1} & L_{M, 2} & \ldots & L_{M, M}
\end{array}\right), \quad \boldsymbol{\rho}:=\left(\begin{array}{c}
\rho_{1} \\
\rho_{2} \\
\vdots \\
\rho_{M}
\end{array}\right), \quad \quad \mathbf{U}^{\mathrm{inc}}:=\left(\begin{array}{c}
\left.u^{\mathrm{inc}}\right|_{\Gamma_{1}} \\
\left.u^{\mathrm{inc}}\right|_{\Gamma_{2}} \\
\vdots \\
\left.u^{\mathrm{inc}}\right|_{\Gamma_{M}}
\end{array}\right) .
$$

The integral formulation (8) provides an exact representation of the wavefields, and in particular of the surface field $\boldsymbol{\rho}$ and the associated far-field pattern (like the Radar Cross Section (RCS)). However, this is at the price of a computationally extensive calculation. Indeed, the linear system (8) is dense due to the nonlocal character of the integral operators. In particular, the size of the system increases thanks to the number of scatterers $M$ when a discretization by a boundary element is applied [39, 50, 51]. This therefore leads to a huge memory requirement as well as a costly numerical solution for the linear system. Clearly, a numerical solution by Krylov subspace iterative solvers [11, 12, 47] can be expected in conjunction with Multilevel Fast Multipole Methods (FMM) [23, 38. Nevertheless, the numerical solution remains expensive to obtain since $M$ can be very large.

\section{OSRCs for single scattering: overview and standard operators}

Because of the high cost of an integral equation solution, new ideas have been developed over the years to find some alternative solutions. Among them, the On-Surface Radiation Condition method, or for the sake of brevity OSRC method, has been introduced in the middle of the 80's by Kriegsmann, Taflove and Umashankar [37. In this paper, the three authors proposed a fast but approximate method for computing the solution to two-dimensional electromagnetic scattering problems by an infinite cylinder with a simple cross section. Extensions and interesting improvements are reported e.g. in [1, 3, 4, 17, 13, 16, 40, 42, 46].

To explain in a synthetic way the OSRC ideas, let us come back to the Helmholtz integral relation (4) also given below, for a single-scattering obstacle $\Omega_{1}=\Omega^{-}$with shape $\Gamma_{1}=\Gamma$,

$$
\forall \mathbf{x} \in \Omega^{+}, \quad u_{1}(\mathbf{x})=-\mathcal{L}_{1}\left(\left.\partial_{\mathbf{n}} u_{1}\right|_{\Gamma_{1}}\right)(\mathbf{x})-\mathcal{M}_{1}\left(\left.u_{1}\right|_{\Gamma_{1}}\right)(\mathbf{x}) .
$$

A simple remark is the following: if we compute the two first traces $\left(\left.u_{1}\right|_{\Gamma_{1}},\left.\partial_{\mathbf{n}_{1}} u_{1}\right|_{\Gamma_{1}}\right)$ of the scattered field (also called Cauchy data $\left(\left.u_{1}\right|_{\Gamma_{1}},\left.\partial_{\mathbf{n}_{1}} u_{1}\right|_{\Gamma_{1}}\right)$ ) we obtain the scattered field from the integral representation (10). This is the heart of the integral equation methods where a suitable exact formulation is built by using the trace relations given in Proposition 3 . In other terms, one wants to get an exact representation of the solution through a boundary operator $D t N_{1}$ linking the trace and the normal derivative trace as

$$
\partial_{\mathbf{n}_{1}} u_{1 \mid \Gamma_{1}}=\operatorname{DtN} N_{1}\left(\left.u_{1}\right|_{\Gamma_{1}}\right), \quad \text { on } \Gamma_{1} .
$$

The operator $D t N_{1}$ is often called the nonlocal Dirichlet-to-Neumann operator. In practice, one way to write the exact (explicit or implicit) representation of the $D t N$ map for a general single 
convex shape $\Gamma=\Gamma_{1}$ is to consider the integral relations. Unfortunately and as previously explained in Section 2, considering integral equations is similar to solving at the discretization level a full linear system. For this reason, Kriegsmann et al. proposed to rather choose an approximation $\Lambda_{1}$ of the $D t N$ operator through a local relation of the following form

$$
\varphi_{1}=\Lambda_{1}\left(\psi_{1}\right), \quad \text { on } \Gamma_{1}
$$

where $\left(\psi_{1}, \varphi_{1}\right)$ is "an approximation" (that we will denote by $\approx$ ) of the exact Cauchy data $\left(\left.u_{1}\right|_{\Gamma_{1}},\left.\partial_{\mathbf{n}_{1}} u_{1}\right|_{\Gamma_{1}}\right)$ and the local approximation $\Lambda_{1}$ of $D t N$ must be explicitly specified. By "local", we mean that we wish to use some differential or partial differential operators over the single surface $\Gamma_{1}$ but not an integral operator. The essential gain is then the fast solution of the associated sparse discrete linear system by using an iterative Krylov subspace method. Finally, a relation like (12) is called an On-Surface Radiation Condition or OSRC.

Let us assume now that we succeeded in deriving such operators. Then, if one considers for example the single-scattering Dirichlet problem, we can set: $\psi_{1}=-\left.u^{\mathrm{inc}}\right|_{\Gamma_{1}}$. As a consequence, the OSRC leads to

$$
\varphi_{1}=-\Lambda_{1}\left(\left.u^{\mathrm{inc}}\right|_{\Gamma_{1}}\right), \quad \text { on } \Gamma_{1} .
$$

Therefore, an approximate value $\varphi_{1}$ of the normal derivative trace is realized by the application of a local operator $\Lambda_{1}$ to the surface field $\left.u^{\mathrm{inc}}\right|_{\Gamma_{1}}$ on the single shape $\Gamma_{1}$. From a practical point of view, this is done by surface finite element methods for a general shape [4, 13] but, as it will be seen in Section 5, special mathematical functions can also be used when a specific shape $\Gamma$ (like a circle) is considered. Reporting this approximation in Equation (10) with $\left(\psi_{1}, \varphi_{1}\right)$ instead of $\left(\left.u_{1}\right|_{\Gamma_{1}},\left.\partial_{\mathbf{n}_{1}} u_{1}\right|_{\Gamma_{1}}\right)$ provides a simple way to compute any valuable physical quantity (like e.g. the far-field pattern).

Among the past years, several kinds of OSRC operators $\Lambda$ have been derived by various authors [3, 4, 7, 13, 16, 40, 42, 46. Here, we propose to focus on some of the most standard operators. Historically, the first class of OSRCs that have been introduced [37] were based on purely local operators. They were built by using absorbing boundary conditions (mainly related to the works by Engquist-Majda [28, and Bayliss-Gunzburger-Turkel [17]). Here, we consider the rigorous version of the half- (for $\ell=1 / 2$ ) and second-order (for $\ell=2$ ) symmetrical OSRCs (see e.g. [6])

$$
\Lambda_{1}^{\ell} \psi_{1}=-\partial_{s_{1}}\left(\alpha_{1}^{\ell} \partial_{s_{1}} \psi_{1}\right)+\beta_{1}^{\ell} \psi_{1}, \quad \text { on } \Gamma_{1},
$$

with

$$
\alpha_{1}^{1 / 2}=0 \quad \text { and } \quad \beta_{1}^{1 / 2}=i k
$$

and

$$
\alpha_{1}^{2}=-\frac{1}{2\left(\kappa_{1}-i k\right)} \quad \text { and } \quad \beta_{1}^{2}=i k-\frac{\kappa_{1}}{2}+\frac{\kappa_{1}^{2}}{8\left(\kappa_{1}-i k\right)} .
$$

In the above relations, $\kappa_{1}$ designates the curvature of $\Gamma_{1}, \partial_{s_{1}}$ is the surface derivative over $\Gamma_{1}$ and the variable $s_{1}$ is the counterclockwise directed surface abscissa over $\Gamma_{1}$.

Even if these OSRCs can be accurate enough in some situations, they are also sometimes limited, implying a need for more precise OSRCs while being still computationally efficient. In the present paper, we consider the square-root and Padé-type boundary conditions developed in [13. The authors propose to rather consider the original square-root operator $\Lambda_{1}^{\sqrt{ }}=i k \sqrt{1+X_{1}}$ for twoand three-dimensional problems setting $X_{1}=\Delta_{\Gamma_{1}} / k^{2}$, where $\Delta_{\Gamma_{1}}$ is the Laplace-Beltrami operator 
over $\Gamma_{1}$. It is shown that the square-root approximation of the total symbol of the $D t N$ map is well-adapted to represent both the propagative and evanescent parts of the scattered field but not the creeping zone. To partially avoid this problem, a regularization of the square-root operator is proposed by including a small damping artificial parameter $\varepsilon_{1}$ to the real wavenumber $k$. In particular, analytical estimates are provided for the complexified wavenumber $k_{\varepsilon_{1}}=k+i \varepsilon_{1}$. The value $\varepsilon_{1}=0.4 k^{2 / 3} \kappa_{1}^{1 / 3}$ is shown to produce very satisfactory results for different model problems even at very high-frequencies. The new OSRC operator

$$
\Lambda_{1}^{\sqrt{ }}=i k \sqrt{1+X_{\varepsilon_{1}}},
$$

with $X_{\varepsilon_{1}}=\partial_{s_{1}}\left(k_{\varepsilon_{1}}^{-2} \partial_{s_{1}} \cdot\right)$, is next localized by using some complex Padé approximants of order $N_{r}$. More precisely, using a rotating branch-cut argument with rotation angle $\theta_{1, r}$ as in [41, the square-root operator is formally approximated by the rational operator

$$
\sqrt{1+X_{1}} \approx C_{0}+\sum_{j=1}^{N_{1, r}} \frac{A_{j} X_{1}}{1+B_{j} X_{1}},
$$

where the complex coefficients $A_{j}, B_{j}$ and $C_{0}$ are explicitly known. Then, a suitable representation of the operator $\Lambda_{1}$ is given through the introduction of auxiliary functions: if $\psi_{1}$ is given, then compute $\varphi_{1}=\Lambda_{1}^{N_{1, r}, \theta_{1, r}} \psi_{1}$ satisfying

$$
\varphi_{1}=i k C_{0} \psi_{1}+i k \sum_{j=1}^{N_{1, r}} \partial_{s_{1}}\left(\frac{A_{j}}{k_{\varepsilon_{1}}^{2}} \partial_{s_{1}} \varphi_{1}^{j}\right),
$$

setting $\varphi_{1}^{j}$ as the solutions to the surface partial differential equations

$$
\partial_{s_{1}}\left(\frac{B_{j}}{k_{\varepsilon_{1}}^{2}} \partial_{s_{1}} \varphi_{1}^{j}\right)+\varphi_{1}^{j}=\psi_{1}, \quad 1 \leq j \leq N_{1, r}
$$

For a general single shape, the implementation of the OSRC methods with Padé approximants can be realized efficiently through a boundary element method [13]. Concerning the square-root operator, its implementation is far from being trivial for a general single scatterer case and may appear as time consuming since the operator is global. In the numerical results considered in this paper, this operator will nevertheless be tested since it can be diagonalized in a Fourier basis when the obstacles are circular cylinders.

\section{OSRCs and multiple scattering for the Dirichlet boundary-value problem}

The goal of this section is to introduce an OSRC formulation for the multiple scattering case. For $M$ obstacles, let us first consider the exact single-layer representation

$$
\forall \mathbf{x} \in \Omega^{+}, u(\mathbf{x})=\mathscr{L} \rho(\mathbf{x})=\sum_{p=1}^{M} u_{p}(\mathbf{x}),
$$

where the elementary scattered field $u_{p}$ by the $p$-th obstacle can be written

$$
u_{p}(\mathbf{x}):=\mathscr{L}_{p}\left(\rho_{p}\right)(\mathbf{x})
$$


where $\rho_{p}:=-\left.\left[\partial_{\mathbf{n}} u\right]\right|_{\Gamma_{p}}$. For each $p, u_{p}$ is outgoing to $\Omega_{p}$ and satisfies the Dirichlet boundary condition

$$
\left.u_{p}\right|_{\Gamma_{p}}:=-\left.u^{\mathrm{inc}}\right|_{\Gamma_{p}}-\left.\sum_{q=1, q \neq p}^{M} u_{q}\right|_{\Gamma_{p}} .
$$

This means that the single scattered fields $u_{q}$ are seen as incident wavefields for $\Omega_{p}$. This is the rigorous integral equations viewpoint.

Now, since we are considering an OSRC approximation, we define an outgoing approximate wavefield $\tilde{u}$ under the form of a single-layer potential through

$$
\forall \mathbf{x} \in \Omega^{+}, \tilde{u}(\mathbf{x})=\mathscr{L} \tilde{\rho}(\mathbf{x})=\sum_{p=1}^{M} \tilde{u}_{p}(\mathbf{x}),
$$

where each OSRC-based generated $p$-th exterior field writes

$$
\tilde{u}_{p}(\mathbf{x}):=\mathscr{L}_{p}\left(\tilde{\rho}_{p}\right)(\mathbf{x})
$$

Here, we search for an approximate normal derivative jump such that $\tilde{\rho}_{q}:=-\varphi_{q}-\left.\partial_{\mathbf{n}} u^{\text {inc }}\right|_{\Gamma_{q}} \approx \rho_{q}$, with $\varphi_{q}$ which also satisfies an $\operatorname{OSRC} \varphi_{q}=\Lambda_{q} \psi_{q}$ on $\Gamma_{q}$, for $1 \leq q \leq M$. Now we force our exterior fields to satisfy the Dirichlet boundary condition

$$
\left.\tilde{u}_{p}\right|_{\Gamma_{p}}:=-\left.u^{\mathrm{inc}}\right|_{\Gamma_{p}}-\left.\sum_{q=1, q \neq p}^{M} \tilde{u}_{q}\right|_{\Gamma_{p}} .
$$

From the trace theorem, one gets, for $q \neq p$,

$$
\left.\tilde{u}_{q}\right|_{\Gamma_{p}}:=L_{p, q} \tilde{\rho}_{q}=-L_{p, q} \varphi_{q}-L_{p, q}\left(\left.\partial_{\mathbf{n}} u^{\mathrm{inc}}\right|_{\Gamma_{q}}\right)=-\left.\left(L_{q} \varphi_{q}\right)\right|_{\Gamma_{p}}-\left(L_{q}\left(\left.\partial_{\mathbf{n}} u^{\mathrm{inc}}\right|_{\Gamma_{q}}\right)\right)_{\mid \Gamma_{p}},
$$

where $L_{p, q} \varphi_{q}=\left.\left(L_{q} \varphi_{q}\right)\right|_{\Gamma_{p}}$. In addition, since $\left.\tilde{u}_{p}\right|_{\Gamma_{p}}$ is supposed to be approximated by $\psi_{p}$, we consider the following trace relation: $\left.\tilde{u}_{p}\right|_{\Gamma_{p}} \approx \psi_{p}$, for $p=1, \ldots, M$. By using this remark in (24), combining with equation (25) and finally by composing by $\Lambda_{p}$, we have the following simplified equation

$$
\varphi_{p}-\sum_{q=1, q \neq p}^{M} \Lambda_{p} L_{p, q} \varphi_{q}=-\Lambda_{p}\left(\left.u^{\mathrm{inc}}\right|_{\Gamma_{p}}\right)+\sum_{q=1, q \neq p}^{M} \Lambda_{p} L_{p, q}\left(\left.\partial_{\mathbf{n}} u^{\mathrm{inc}}\right|_{\Gamma_{q}}\right), \quad \text { on } \Gamma_{p} .
$$

An alternative way of writing the above system is

$$
\left(\begin{array}{cccc}
I_{1,1} & -\Lambda_{1} L_{1,2} & \ldots & -\Lambda_{1} L_{1, M} \\
-\Lambda_{2} L_{2,1} & I_{2,2} & \ldots & -\Lambda_{2} L_{2, M} \\
\vdots & \vdots & \ddots & \vdots \\
-\Lambda_{M} L_{M, 1} & -\Lambda_{M} L_{M, 2} & \cdots & I_{M, M}
\end{array}\right)\left(\begin{array}{c}
\varphi_{1} \\
\varphi_{2} \\
\vdots \\
\varphi_{M}
\end{array}\right)=\Psi^{\text {inc }}
$$

where the right hand side is given by

$$
\Psi^{\mathrm{inc}}=-\left(\begin{array}{c}
\left.\Lambda_{1} u^{\mathrm{inc}}\right|_{\Gamma_{1}} \\
\left.\Lambda_{2} u^{\mathrm{inc}}\right|_{\Gamma_{2}} \\
\vdots \\
\left.\Lambda_{M} u^{\mathrm{inc}}\right|_{\Gamma_{M}}
\end{array}\right)+\left(\begin{array}{cccc}
0_{1,1} & \Lambda_{1} L_{1,2} & \ldots & \Lambda_{1} L_{1, M} \\
\Lambda_{2} L_{2,1} & 0_{2,2} & \ldots & \Lambda_{2} L_{2, M} \\
\vdots & \vdots & \ddots & \vdots \\
\Lambda_{M} L_{M, 1} & \Lambda_{M} L_{M, 2} & \ldots & 0_{M, M}
\end{array}\right)\left(\begin{array}{c}
\left.\partial_{\mathbf{n}} u^{\mathrm{inc}}\right|_{\Gamma_{1}} \\
\left.\partial_{\mathbf{n}} u^{\mathrm{inc}}\right|_{\Gamma_{2}} \\
\vdots \\
\left.\partial_{\mathbf{n}} u^{\mathrm{inc}}\right|_{\Gamma_{M}}
\end{array}\right) .
$$


If we define the operators

$$
\mathcal{I}:=\operatorname{diag}\left(\left(I_{j, j}\right)_{j=1, \ldots, M}\right), \quad \mathcal{L}_{\Lambda}=\left(\begin{array}{cccc}
0_{1,1} & \Lambda_{1} L_{1,2} & \ldots & \Lambda_{1} L_{1, M} \\
\Lambda_{2} L_{2,1} & 0_{2,2} & \ldots & \Lambda_{2} L_{2, M} \\
\vdots & \vdots & \ddots & \vdots \\
\Lambda_{M} L_{M, 1} & \Lambda_{M} L_{M, 2} & \ldots & 0_{M, M}
\end{array}\right)
$$

and

$$
\Lambda:=\left(\begin{array}{c}
\Lambda_{1} \\
\Lambda_{2} \\
\vdots \\
\Lambda_{M}
\end{array}\right), \quad \partial_{\mathbf{n}} \mathbf{U}^{\mathrm{inc}}:=\left(\begin{array}{c}
\left.\partial_{\mathbf{n}} u^{\mathrm{inc}}\right|_{\Gamma_{1}} \\
\left.\partial_{\mathbf{n}} u^{\mathrm{inc}}\right|_{\Gamma_{2}} \\
\vdots \\
\left.\partial_{\mathbf{n}} u^{\mathrm{inc}}\right|_{\Gamma_{M}}
\end{array}\right)
$$

then system (27) reads

$$
\left(\mathcal{I}-\mathcal{L}_{\Lambda}\right) \varphi=\Psi^{\text {inc }}:=-\Lambda \cdot \mathbf{U}^{\text {inc }}+\mathcal{L}_{\Lambda} \partial_{\mathbf{n}} \mathbf{U}^{\text {inc }}
$$

A nicer and equivalent writing of this last equation is

$$
\left(\mathcal{I}-\mathcal{L}_{\Lambda}\right) \tilde{\boldsymbol{\rho}}=-\left(\partial_{\mathbf{n}} \mathbf{U}^{\mathrm{inc}}-\Lambda \cdot \mathbf{U}^{\mathrm{inc}}\right)
$$

Let us remark that system (32) is defined by a second-kind integral equation with the perturbed smoothing operator $\mathcal{L}_{\Lambda}$. This means that its numerical solution through an iterative solver (e.g. iterative Krylov subspace solver) should be particularly efficient since it exhibits a fast convergence rate due to the distribution of its eigenvalues in the complex plane.

\section{The example of scattering by a cluster of circular cylinders}

\subsection{Numerical approximation for a cluster of circular cylinders}

Let us now analyze the numerical validity of the OSRC approach for solving the Dirichlet multiple scattering problem. Our goal here is to understand the quality of the mathematical approximate OSRC formulations in several situations. To this end and to not introduce accuracy problems that could be related to the fact that a boundary element method is used, we consider the special case where the scatterers are all circular cylinders. The situation with more general objects will be analyzed in a forthcoming paper. For circular cylinders, a Mie series expansion of the solutions can be derived which allows to measure the "continuous" accuracy of the OSRC formulation. Indeed, almost no numerical error related to the numerical approximation by Mie series expansions arises since the method is spectrally accurate [9, 51. Most particularly, the choice of the OSRC operator is of course crucial for the approximation quality and is investigated below.

To implement the following examples, we use the $\mu$-diff Matlab toolbox 1 [51] that has been developed for solving scattering problems with integral formulations and for complex multiple scattering configurations involving circular cylinders. In $\mu$-diff, the numerical solution based on the EFIE formulation (8) is already available. Concerning the OSRC formulation (32), the numerical

\footnotetext{
${ }^{1}$ http://mu-diff.math.cnrs.fr/mu-diff/
} 
approximation by a Mie series expansion can also be obtained easily. Indeed, this formulation mainly considers integral operators that can be written directly in $\mu$-diff. In addition, the OSRC operators introduced in section 3 can all be diagonalized in the Fourier basis. This can be made mode-by-mode since the angular derivative $\partial_{\theta}$ corresponds to a multiplication by the Fourier symbol ( $m$ for $\mu$-diff, see [51]). The application of an OSRC operator can then be reduced to multiplying by a diagonal matrix when the Mie series expansion is truncated. We do not give the details of the implementation here but rather analyze the numerical results. We first start by considering a simple scattering problem in section 5.2 involving two disks. Then, in sections 5.3 and 5.4 , we investigate the case of scattering by a triangular lattice and next a cluster of randomly distributed circular cylinders.

In the sequel, we use the following notations [9, 51]. The scattering obstacle $\Omega^{-}$is the union of $M$ disks $\Omega_{p}^{-}$, for $p=1, \ldots, M$, of radius $a_{p}$ and center $\mathbf{O}_{p}$. For any $p, q=1, \ldots, M$, with $q \neq p$, we introduce $\mathbf{b}_{p q}=\mathbf{O}_{q} \mathbf{O}_{p}$ as the vector between the centers $\mathbf{O}_{q}$ and $\mathbf{O}_{p}$ as the distance between two centers $b_{p q}=\left\|\mathbf{b}_{p q}\right\|$.

\subsection{Example 1: scattering by two circular cylinders}

The first test case considers two circular cylinders $\Omega_{1}^{-}$and $\Omega_{2}^{-}$, with $\mathbf{O}_{2}=(b / 2,0)=-\mathbf{O}_{1}$ (with $b>0$ ), which means that $b_{12}=b$. In the notations, the OSRC formulation for multiple scattering with the OSRC (14)-(15) for single scattering is denoted by OSRC ${ }^{1 / 2}$, OSRC ${ }^{2}$ with (14)(16), OSRC $\sqrt{ }$ with $(17)$ and finally $\operatorname{OSRC}^{N_{r}, \theta_{r}}$ for $(17)$ but where the square-root is approximated by the Padé expansion (18). For this last case, we fix the standard values of the parameters to $N_{r}=N_{p, r}=4$ and $\theta_{r}=\theta_{p, r}=\pi / 4$, for $p=1, \ldots, M$, (see e.g. [13]). Since there is no noticeable difference numerically between $\mathrm{OSRC}^{N_{r}, \theta_{r}}$ and OSRC $\sqrt{ }$, we essentially report only the results for OSRC $^{N_{r}, \theta_{r}}$ (which corresponds to a practical OSRC computation [13]). The incident fields are chosen as the plane wave $u^{\text {inc }}(\mathbf{x})=e^{i k \boldsymbol{\alpha} \cdot \mathbf{x}}$, with an incidence direction $\boldsymbol{\alpha}=(\cos (\alpha), \sin (\alpha))$.

Let us start by studying a low-frequency problem. We fix $a_{1}=a_{2}=a=0.5, b=1.2$ and $k a=0.5(k=1)$. The angle of incidence is $\alpha=0$. For this situation, OSRC $^{1 / 2}$ does not provide some correct numerical results. This can be seen on Figure 1(b) where we represent the scattered field by the two objects, compared to the reference solution 1(a), More computations show that this is true whatever is the incidence angle. This is quite well-known from single-scattering since the simple associated OSRC operator is not accurate enough at low-frequency. The accuracy is higher when OSRC $^{2}$ is used, due to the corrective terms that appear in the boundary operator. The associated scattered field is reported on 1(c). Now if one considers $\operatorname{OSRC}^{N_{r}, \theta_{r}}$ (as well as the square-root version OSRC $\sqrt{ }$ ), then a loss of accuracy can be observed (see Figure $1(\mathrm{~d})$. The precision can be recovered if one chooses the following new square-root OSRC with second-order terms correction (denoted by $\left.\mathrm{OSRC}{ }^{\prime 2}\right)$ for the $p$-th scatterer $(p=1, \ldots, M)$

$$
\Lambda_{p}^{\sqrt{ }, 2}=i k \sqrt{1+X_{\varepsilon_{p}}}+\left(\beta_{p}^{2}-i k\right)
$$

with $\beta_{p}^{2}$ given by

$$
\beta_{p}^{2}=i k-\frac{\kappa_{p}}{2}+\frac{\kappa_{p}^{2}}{8\left(\kappa_{p}-i k\right)}
$$

setting $\kappa_{p}=1 / a_{p}$. The associated Padé approximation is denoted by $\operatorname{OSRC}^{N_{r}, \theta_{r}, 2}$. The results are reported on Figure 1(e). In addition, we observe that the Radar Cross Section (RCS) defined 
by (see e.g. [9, 51])

$$
\forall \theta \in[0,2 \pi], \quad \operatorname{RCS}(\theta)=10 \log _{10}\left(2 \pi|\mathcal{A}(\theta)|^{2}\right)(\mathrm{dB}),
$$

is also accurate (Figure $1(\mathrm{f})$, setting as $\mathcal{A}$ the scattering amplitude. For much lower frequencies $(k \leq 0.1)$, the accuracy is not satisfactory. Alternative OSRCs might then be considered [40, 45] for single-scattering, improving hence the accuracy of the global method for the very low-frequency multiple scattering situation.

One reason why the OSRC can have a loss of accuracy is related to the fact that the computations in the shadow zone are partially included into the OSRC representation (see [13]). Let us first consider that the incidence angle is now set to $\alpha=\pi / 2$ (for the same parameters as in the previous case). Then, one can see that the reference (Figure 2(a) and $\mathrm{OSRC}^{N_{r}, \theta_{r}, 2}$ solutions (Figure 2(b)) are very similar. Now, we let $\alpha=0$ but consider a larger separation distance $b=4$. We can remark that the results (see Figures $3(\mathrm{a})(\mathrm{b})(\mathrm{c})$ ) are yet correct which means that for the low frequency regime, the creeping rays are less present into the reference solution.

Let us investigate now the higher frequency regime. The first case considers $k a=2.5$ with the parameters $a_{1}=a_{2}=a=0.5, k=5$ and $b=1.2$, for $\alpha=0$. We only present on Figures (4) the numerical results for the most accurate OSRC, that is $\mathrm{OSRC}^{N_{r}, \theta_{r}, 2}$. We observe that the computations are correct but depending on the scattering angle. This is related to the fact that the incidence angle is zero and that the shadow zone is significant (compare Figures 4(a) and 4(b), and the corresponding RCS curves on Figure 4(c) ). This is much improved according to the incidence angle (see Figures 5(a) and 5(b) for $\alpha=\pi / 4$ and $b=1.2$, the other parameters being the same) or to the distance between the scatterers (see Figures 6(a) 6(b) and 6(c) for $\alpha=0$ and $b=4$ ).

Finally, we report some results for a much higher frequency, i.e. $k=30$, for the same scatterers with $b=1.2$, and two scattering angles, $\alpha=0$ (Figures 7) and $\alpha=\pi / 4$ (Figures 8). We see that, according to the situation, the results can be more or less precise for the scattered field and the RCS, confirming then the previous results.

\subsection{Example 2: scattering by a triangular lattice of circular cylinders}

The triangular lattice is a structure that is composed of two parallel horizontal single-rows. The first one contains $M_{x} \geq 2$ equally spaced disks and the second one $M_{x}-1$. The horizontal distance between two objects is $b_{x}$. Next, the first row is repeated vertically $M_{y}$ times with a uniform distance $b_{y}=b_{1\left(2 M_{x}\right)}$, while the second one is reproduced $M_{y} \pm 1$ times with again a separation distance $b_{y}$ (see e. g. Figure 9(a)p.

The first numerical example reported on Figures 9 considers the values $M_{x}=10$ and $M_{y}=10$, for a total of 95 circular cylinders. The separation distances are fixed to $b_{x}=b_{y}=0.8$. The radii are equal to $a=0.2$ and the wavenumber is $k=2$ for an incidence angle $\alpha=\pi / 6$. The second example considers the same situation but for the larger wavenumber $k=15$ (see Figures 10 ). In both cases, the RCS results are correct even if a loss of accuracy can be observed for higher frequencies since the incident wave penetrates deeper inside the cluster for larger $k$ and then more multiple scattering effects take place. They are partially reproduced through the OSRC approximation.

\subsection{Example 3: scattering by randomly distributed circular cylinders}

The first case considers $M=120$ randomly distributed circular cylinders with radii $a_{p}$ such that: $0.18 \leq a_{p} \leq 0.22$, for $p=1, \ldots, M$ (see Figure 11(a) $)$. They are all set in the domain $]-5 ; 5\left[^{2}\right.$. The minimal distance between the scatterers is fixed to 0.1 . The plane wave is incident at $\alpha=\pi / 6$ 
for $k=2$. The RCS results are reported on Figure 11(b), We observe that the accuracy is correct. This can be explained from Figures $11(\mathrm{c})$ 11(d) by the fact that the scattered field outside the cluster is well-reproduced even if some inaccuracies arise inside.

For the last test-case, we have $M=100$ randomly distributed circular cylinders (see Figure $12(\mathrm{a})$ with some radii $a_{p}$ that satisfy $0.18 \leq a_{p} \leq 0.22$, for $p=1, \ldots, M$. They are placed inside the box $]-5 ; 5\left[^{2}\right.$. The minimal distance between two scatterers is 0.1 . We consider $k=8$ for an incidence angle $\alpha=\pi / 4$. One can see on Figures $12(\mathrm{c})-12(\mathrm{~d})$ that the scattered field is extremely complex to reproduce due to the strong penetration of the incident wave inside the cluster of circular cylinders. However, the RCS remains acceptable even if the precision of the amplitude at some scattering angles is sometimes not correct (see Figure 12(b)).

\section{Conclusion}

This paper introduced an extension of the OSRC method to multiple scattering problems by a cluster of cylinders. The method has the advantage of being generic and can consider well-adapted OSRCs with respect to the frequency regime under investigation. The formulation is tested for several cases involving circular cylinders, from the low- to the high-frequency regime. This shows that the method can lead to relatively accurate computations of the scattered field and associated far-field.

This work is a first step towards the development of more efficient algorithms [8] based on the OSRC method for arbitrarily shaped two- and three-dimensional obstacles, with the possibility of handling more complex boundary conditions. This reliable numerical solution would be able to provide a fast (iterative) algorithm for multiple scattering but could also lead to efficient preconditioners for the full solution by integral equations, in the spirit of the ideas investigated in [10, 11]. These questions will be addressed in some future works.

Acknowledgments. This work has been funded by the Institute of Scientific Research and Revival of Islamic Heritage at Umm Al-Qura University (project ID 43405027).

\section{References}

[1] S. Acosta. On-surface radiation condition for multiple scattering of waves. Comput. Meth. Appl. Mech. Engrg., 283(1296-1309), 2015.

[2] S. Acosta and V. Villamizar. Coupling of Dirichlet-to-Neumann boundary condition and finite difference methods in curvilinear coordinates for multiple scattering. J. Comput. Phys., 229(5498-5517), 2010.

[3] H. Ammari. Scattering of waves by thin periodic layers at high frequencies using the on-surface radiation condition method. IMA J. Appl. Math., 60:199-215, 1997.

[4] X. Antoine. Fast approximate computation of a time-harmonic scattered field using the onsurface radiation condition method. IMA J. Appl. Math., 66(1):83-110, 2001.

[5] X Antoine. An algorithm coupling the OSRC and FEM for the computation of an approximate scattered acoustic field by a non-convex body. International Journal for Numerical Methods in Engineering, 54(7):1021-1041, 2002. 
[6] X. Antoine, H. Barucq, and A. Bendali. Bayliss-Turkel-like radiation conditions on surfaces of arbitrary shape. J. Math. Anal. Appl., 229(1):184-211, 1999.

[7] X. Antoine, A. Bendali, and M. Darbas. Analytic preconditioners for the boundary integral solution of the scattering of acoustic waves by open surfaces. J. Comput. Acoust., 3(13):477498, 2005.

[8] X Antoine, C. Chniti, and S. Eisa Ali Alhazmi. Computational methods based on OSRC techniques for solving multiple acoustic scattering problems. in preparation, 2016.

[9] X. Antoine, C. Chniti, and K. Ramdani. On the numerical approximation of high-frequency acoustic multiple scattering problems by circular cylinders. J. Comput. Phys., 227(3):17541771, 2008.

[10] X. Antoine and M. Darbas. Alternative integral equations for the iterative solution of acoustic scattering problems. Quaterly J. Mech. Appl. Math., 1(58):107-128, 2005.

[11] X. Antoine and M. Darbas. Generalized combined field integral equations for the iterative solution of the three-dimensional Helmholtz equation. M2AN Math. Model. Numer. Anal., $1(41): 147-167,2007$.

[12] X. Antoine and M. Darbas. Integral Equations and Iterative Schemes for Acoustic Scattering Problems. to appear, 2015.

[13] X. Antoine, M. Darbas, and Y.Y. Lu. An improved surface radiation condition for highfrequency acoustic scattering problems. Comput. Methods Appl. Mech. Engrg., 195(3336):4060-4074, 2006.

[14] X. Antoine, C. Geuzaine, and K. Ramdani. Wave Propagation in Periodic Media - Analysis, Numerical Techniques and Practical Applications, volume 1, chapter Computational Methods for Multiple Scattering at High Frequency with Applications to Periodic Structures Calculations, pages 73-107. Progress in Computational Physics, 2010.

[15] X. Antoine, K. Ramdani, and B. Thierry. Wide frequency band numerical approaches for multiple scattering problems by disks. J. Algorithms Comput. Technol., 6(2):241-259, 2012.

[16] A. Atle and B. Engquist. On surface radiation conditions for high-frequency wave scattering. J. Comp. Appl. Math., 204(2):306 - 316, 2007. Special Issue: The Seventh International Conference on Mathematical and Numerical Aspects of Waves (WAVES1705).

[17] A. Bayliss, M. Gunzburger, and E. Turkel. Boundary conditions for the numerical solution of elliptic equations in exterior regions. SIAM J. Appl. Math., 42(2):430-451, 1982.

[18] S. Bidault, F.J.G. de Abajo, and A. Polman. Plasmon-based nanolenses assembled on a welldefined DNA template. Journal of the American Chemical Society, 130(9):2750+, 2008.

[19] H. Brakhage and P. Werner. Über das Dirichletsche Aussenraumproblem für die Helmholtzsche Schwingungsgleichung. Arch. Math., 16:325-329, 1965. 
[20] J.T. Chen, Y.T. Lee, Y.J. Lin, I.L. Chen, and J.W. Lee. Scattering of sound from point sources by multiple circular cylinders using addition theorem and superposition technique. Numerical Methods for Partial Differential Equations, 27(1365-1383), 2011.

[21] D. Colton and R. Kress. Inverse Acoustic and Electromagnetic Scattering Theory, volume 93 of Applied Mathematical Sciences. Springer-Verlag, Berlin, second edition, 1998.

[22] D. L. Colton and R. Kress. Integral Equation Methods in Scattering Theory. Pure and Applied Mathematics (New York). John Wiley \& Sons Inc., New York, 1983. A Wiley-Interscience Publication.

[23] E. Darve. The fast multipole method: numerical implementation. J. Comput. Phys., 160(1):195-240, 2000.

[24] T.E. Doyle, D.A. Robinson, S.B. Jones, K.H. Warnick, and B.L. Carruth. Modeling the permittivity of two-phase media containing monodisperse spheres: Effects of microstructure and multiple scattering. Physical Review B, 76(5), 2007.

[25] T.E. Doyle, A.T. Tew, K.H. Warnick, and B.L. Carruth. Simulation of elastic wave scattering in cells and tissues at the microscopic level. Journal of the Acoustical Society of America, 125(3):1751-1767, 2009.

[26] M. Ehrhardt. Wave Propagation in Periodic Media Analysis, Numerical Techniques and practical Applications, E-Book Series Progress in Computational Physics (PiCP), Volume 1. Bentham Science Publishers, 2010.

[27] M. Ehrhardt, H. Han, and C. Zheng. Numerical simulation of waves in periodic structures. Commun. Comput. Phys., 5:849-870, 2009.

[28] B. Engquist and A. Majda. Absorbing boundary conditions for the numerical simulation of waves. Math. Comp., 31(139):629-651, 1977.

[29] P. Ferrand, J. Wenger, A. Devilez, M. Pianta, B. Stout, N. Bonod, E. Popov, and H. Rigneault. Direct imaging of photonic nanojets. Optics Express, 16(10):6930-6940, 2008.

[30] C. Geuzaine, O. Bruno, and F. Reitich. On the $\mathrm{O}(1)$ solution of multiple-scattering problems. IEEE Trans. Magn., 41(5):1488-1491, May 2005. 11th IEEE Biennial Conference on Electromagnetic Field Computation, Seoul, South Korea, June 06-09, 2004.

[31] L. Greengard and V. Rokhlin. A fast algorithm for particle simulations. J. Comput. Phys., $73(2): 325-348,1987$.

[32] M.J. Grote and C. Kirsch. Dirichlet-to-Neumann boundary conditions for multiple scattering problems. J. Comput. Phys., 201(2):630 - 650, 2004.

[33] R.F. Harrington and J.R. Mautz. H-field, E-field and combined field solution for conducting bodies of revolution. Archiv Elektronik und Uebertragungstechnik, 4(32):157-164, 1978.

[34] Z. Hu and Y.Y. Lu. Compact wavelength demultiplexer via photonic crystal multimode resonators. J. Opt. Soc. Amer. B, to appear 2014. 
[35] R.D. Meade J.D. Joannopoulos and J.N. Winn. Photonic Crystals: Molding the Flow of Light. Princeton University Press, 1995.

[36] A.A. Kharlamov and P. Filip. Generalisation of the method of images for the calculation of inviscid potential flow past several arbitrarily moving parallel circular cylinders. Journal of Engineering Mathematics, 77(1), 2012.

[37] G.A. Kriegsmann, A. Taflove, and K.R. Umashankar. A new formulation of electromagnetic wave scattering using an on-surface radiation boundary condition approach. IEEE Trans. Antennas and Propagation, 35(2):153-161, 1987.

[38] Y. Liu. Fast Multipole Boundary Element Method. Theory and Applications in Engineering. Cambridge University Press, 2009.

[39] P. A. Martin. Multiple Scattering. Interaction of Time-Harmonic Waves with N Obstacles, volume 107 of Encyclopedia of Mathematics and its Applications. Cambridge University Press, Cambridge, 2006.

[40] M. Medvinsky and E. Turkel. On surface radiation conditions for an ellipse. J. Comput. Appl. Math., 234(6):1647-1655, 2010.

[41] FA Milinazzo, CA Zala, and GH Brooke. Rational square-root approximations for parabolic equation algorithms. Journal of the Acoustical Society of America, 101(2):760-766, 1997.

[42] R.D. Murch. The on-surface radiation condition applied to three-dimensional convex objects. IEEE Trans. Antennas and Propagation, 41(5):651-658, 1993.

[43] D.M. Natarov, V.O. Byelobrov, R. Sauleau, T.M. Benson, and A.I. Nosich. Periodicity-induced effects in the scattering and absorption of light by infinite and finite gratings of circular silver nanowires. Optics Express, 19(22176-22190), 2011.

[44] J.-C. Nédélec. Acoustic and Electromagnetic Equations. Integral Representations for Harmonic Problems, volume 144 of Applied Mathematical Sciences. Springer-Verlag, New York, 2001.

[45] R. Reiner, R. Djellouli, and I. Harari. The performance of local absorbing boundary conditions for acoustic scattering from elliptical shapes. Comput. Methods Appl. Mech. Engrg., 195(2932):3622-3665, 2006.

[46] R.G. Roxburgh. Electromagnetic scattering from a right-circular cylinder using a surface radiation condition. IMA J. Appl. Math., 59:221-230, 1997.

[47] Y. Saad. Iterative Methods for Sparse Linear Systems. SIAM, (2nd edition) 2003.

[48] R. Savo, M. Burresi, T. Svensson, K. Vynck, and D.S. Wiersma. Walk dimension for light in complex disordered media. Phys. Rev. A, 90:023839, Aug 2014.

[49] B. Thierry. Analyse et Simulations Numériques du Retournement Temporel et de la Diffraction Multiple. Nancy University, Thèse de Doctorat, 2011.

[50] B. Thierry. A remark on the single scattering preconditioner applied to boundary integral equations. Journal of Mathematical Analysis and Applications, 413(1):212 - 228, 2014. 
[51] B. Thierry, X. Antoine, C. Chniti, and H. Alzubaidi. $\mu$-diff: An open-source Matlab toolbox for computing multiple scattering problems by disks. Computer Physics Communications, 192:348-362, 2015.

[52] L. Tsang, J.A. Kong, K.H. Ding, and C.O. Ao. Scattering of Electromagnetic Waves, Numerical Simulation. Wiley Series in Remote Sensing. J.A. Kong, Series Editor, 2001.

[53] B. Van Genechten, B. Bergen, D. Vandepitte, and W. Desmet. A Trefftz-based numerical modelling framework for Helmholtz problems with complex multiple-scatterer configurations. J. Comput. Phys., 229(6623-6643), 2010.

[54] D.S. Wiersma. Disordered photonics. Nature Photonics, 7:188-196, Feb 2013. 


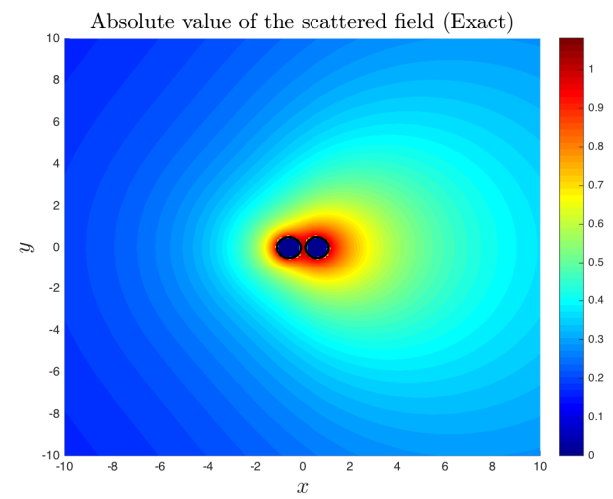

(a) Reference solution

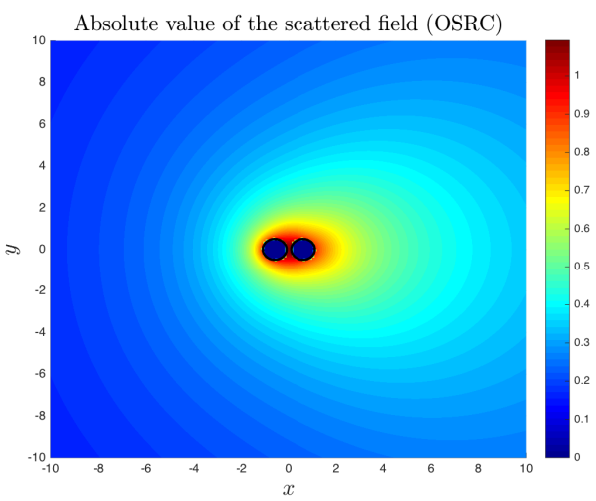

(c) OSRC $^{2}$ solution

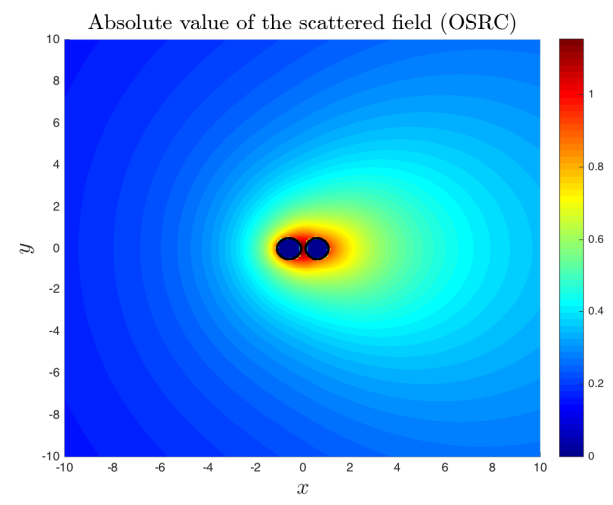

(e) $\operatorname{OSRC}^{N_{r}, \theta_{r}, 2}$ solution

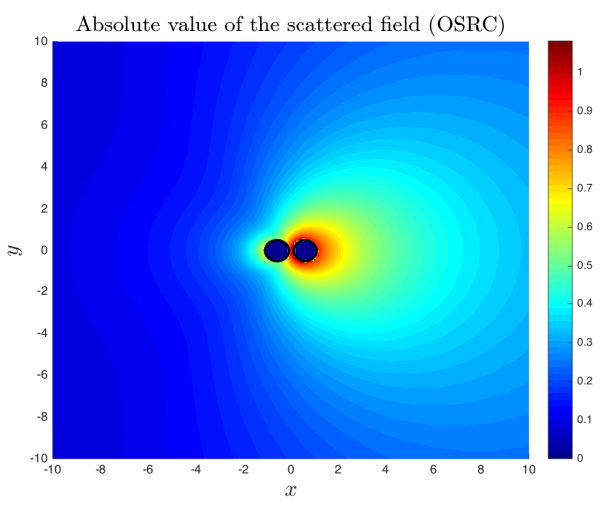

(b) $\mathrm{OSRC}^{1 / 2}$ solution

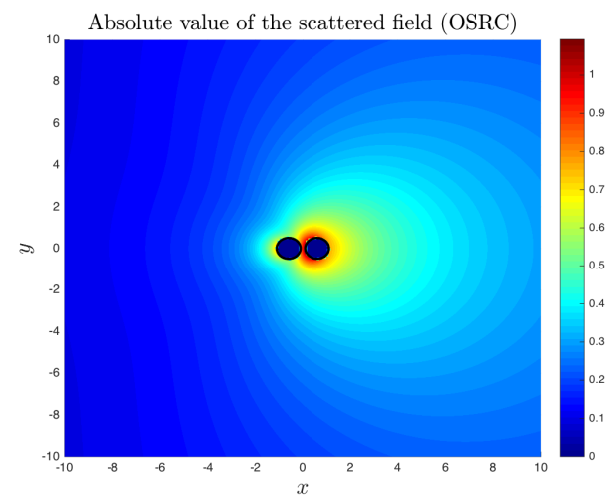

(d) $\operatorname{OSRC}^{N_{r}, \theta_{r}}$ solution

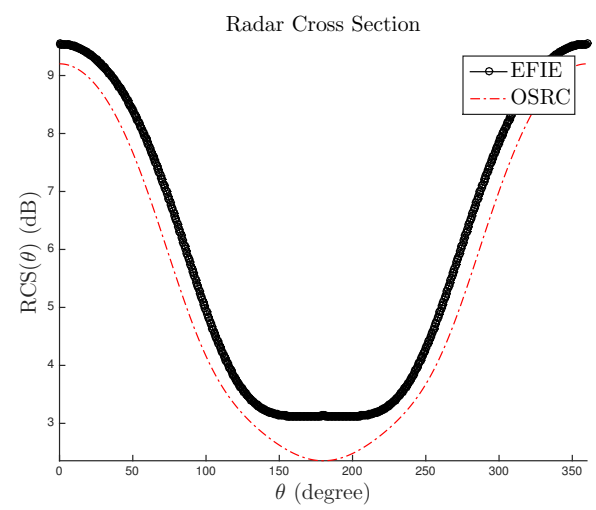

(f) RCS comparison

Figure 1: Scattering by two circular cylinders: comparison of the amplitude of the scattered fields for (a): the reference solution and the four OSRC solutions ((b): OSRC ${ }^{1 / 2},(\mathrm{c})$ : $\operatorname{OSRC}^{2}$ and (d:) $\operatorname{OSRC}^{N_{r}, \theta_{r}},(\mathrm{e})$ : $\operatorname{OSRC}^{N_{r}, \theta_{r}, 2}$ ). Furthermore, (f) reports the RCS for the reference solution and $\operatorname{OSRC}^{N_{r}, \theta_{r}, 2}$. The chosen parameters are: $a_{1}=a_{2}=$ $a=0.5$, and $b=1.2$. The wavenumber $k$ is such that $k a=0.5$ and the angle of incidence is $\alpha=0$. 


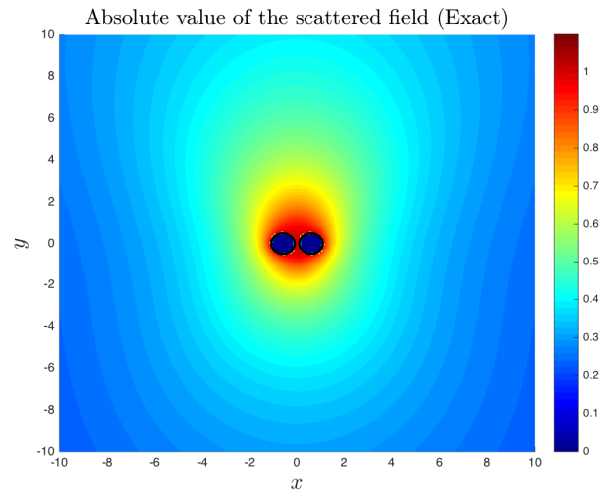

(a) Reference solution

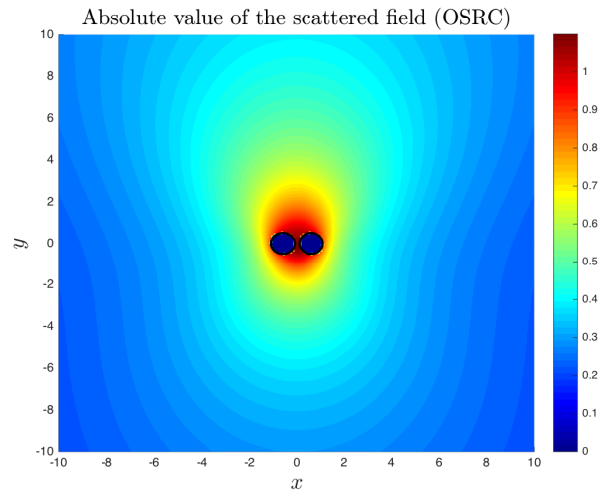

(b) $\operatorname{OSRC}^{N_{r}, \theta_{r}, 2}$ solution

Figure 2: Scattering by two circular cylinders: comparison of the amplitude of the scattered fields for (a): the reference solution and (b): $\operatorname{OSRC}^{N_{r}, \theta_{r}, 2}$. The chosen parameters are: $a_{1}=a_{2}=a=0.5$, and $b=1.2$. The wavenumber $k$ is such that $k a=0.5$ and the angle of incidence is $\alpha=\pi / 2$. 


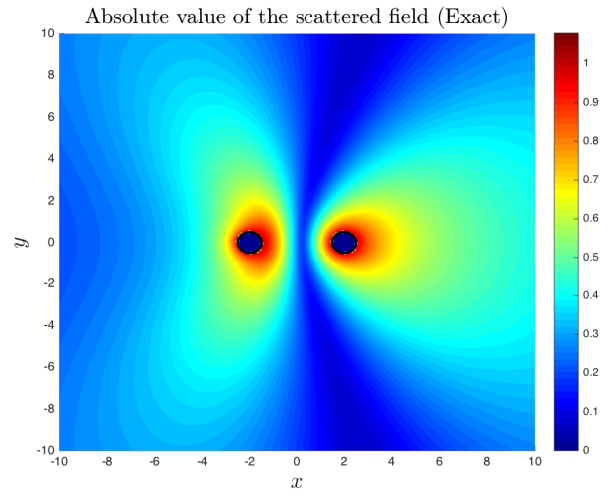

(a) Reference solution

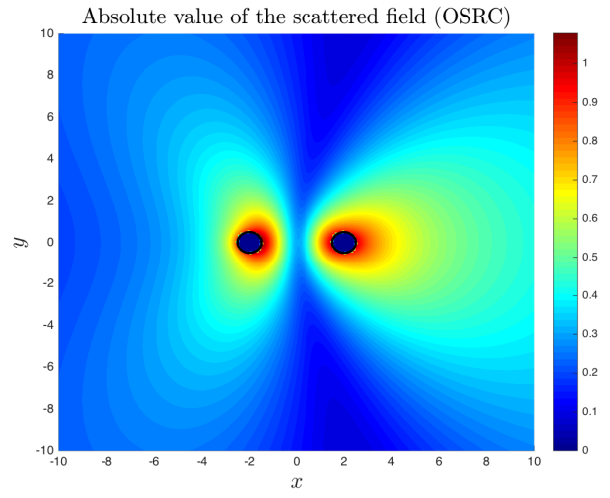

(b) $\operatorname{OSRC}^{N_{r}, \theta_{r}, 2}$ solution

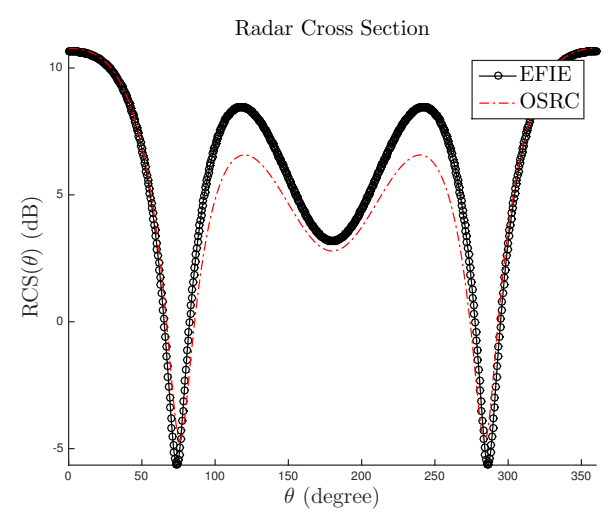

(c) RCS comparison

Figure 3: Scattering by two circular cylinders: comparison of the amplitude of the scattered fields for (a): the reference solution and (b): $\mathrm{OSRC}^{N_{r}, \theta_{r}, 2}$. The RCS are reported on Figure (c). The chosen parameters are: $a_{1}=a_{2}=a=0.5$, and $b=4$. The wavenumber $k$ is such that $k a=0.5$ and the angle of incidence is $\alpha=0$. 


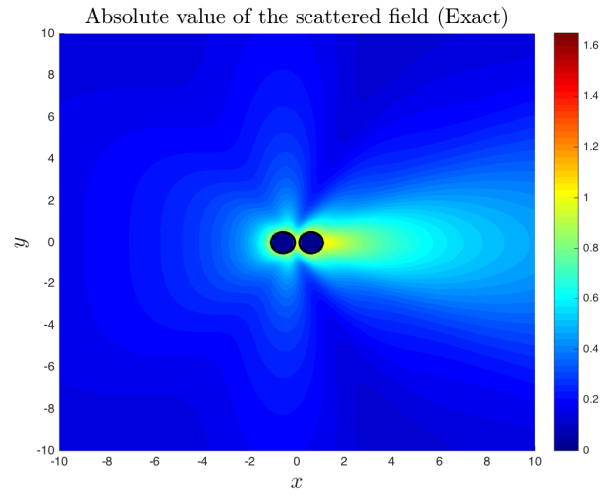

(a) Reference solution

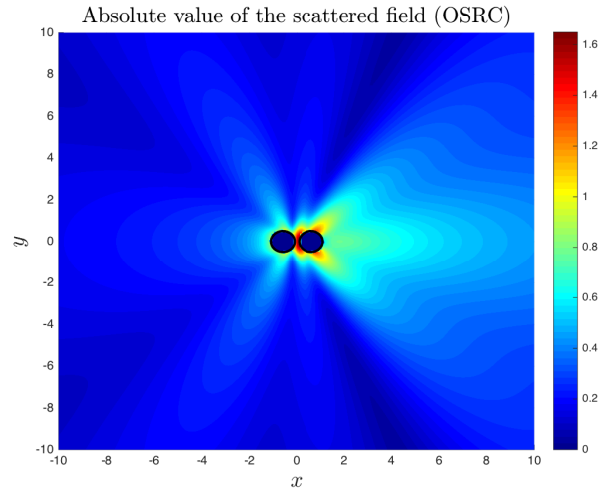

(b) $\operatorname{OSRC}^{N_{r}, \theta_{r}, 2}$ solution

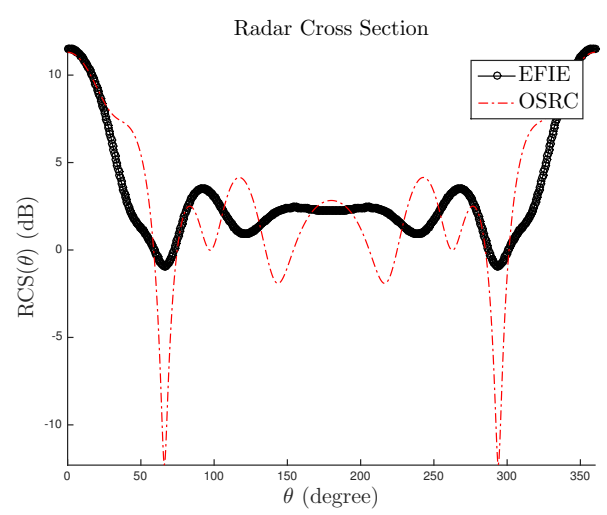

(c) RCS comparison

Figure 4: Scattering by two circular cylinders: comparison of the amplitude of the scattered fields for (a): the reference solution and (b): $\operatorname{OSRC}^{N_{r}, \theta_{r}, 2}$. The RCS are reported on Figure (c) The chosen parameters are: $a_{1}=a_{2}=a=0.5$, and $b=1.2$. The wavenumber $k$ is such that $k a=2.5$ and the angle of incidence is $\alpha=0$. 


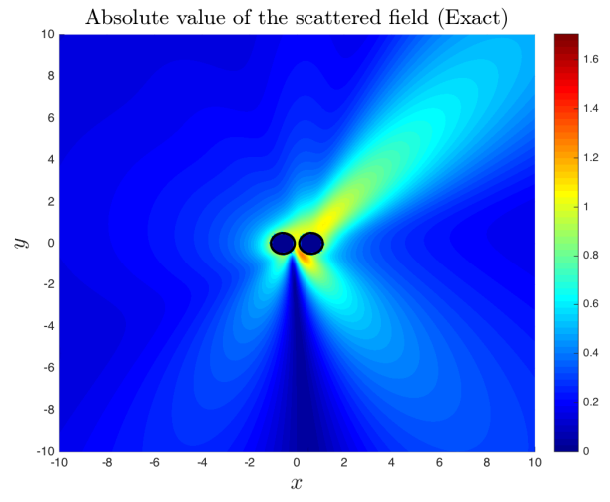

(a) Reference solution

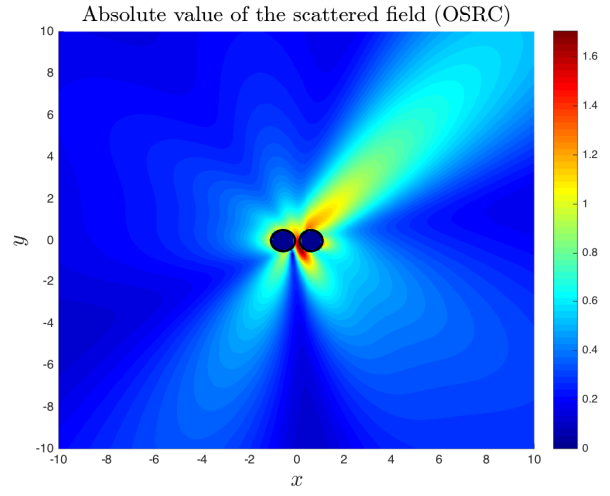

(b) $\operatorname{OSRC}^{N_{r}, \theta_{r}, 2}$ solution

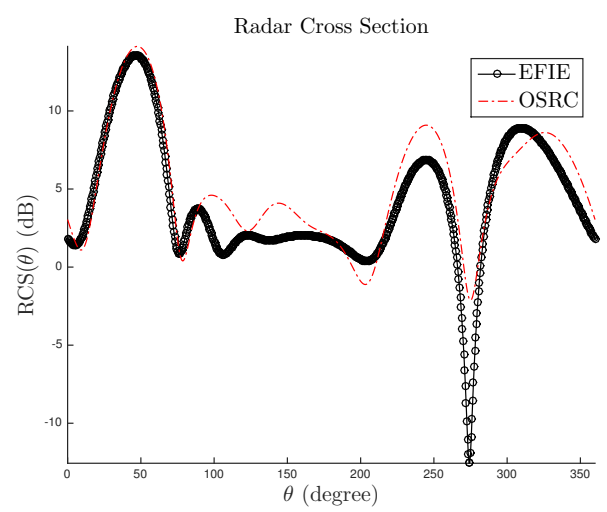

(c) RCS comparison

Figure 5: Scattering by two circular cylinders: comparison of the amplitude of the scattered fields for (a): the reference solution and (b): $\mathrm{OSRC}^{N_{r}, \theta_{r}, 2}$. The RCS are reported on Figure (c). The chosen parameters are: $a_{1}=a_{2}=a=0.5$, and $b=1.2$. The wavenumber $k$ is such that $k a=2.5$ and the angle of incidence is $\alpha=\pi / 4$. 


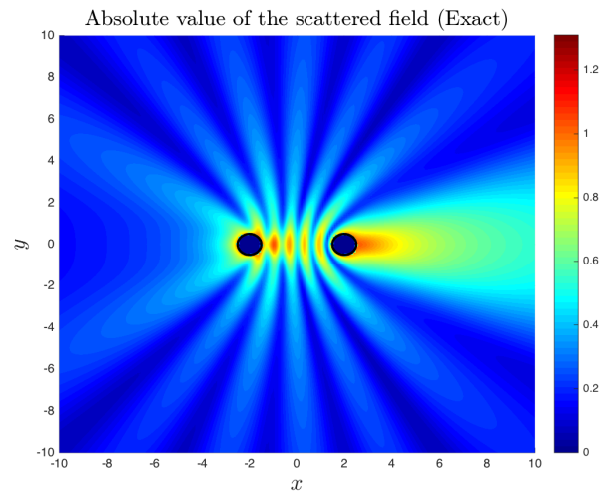

(a) Reference solution

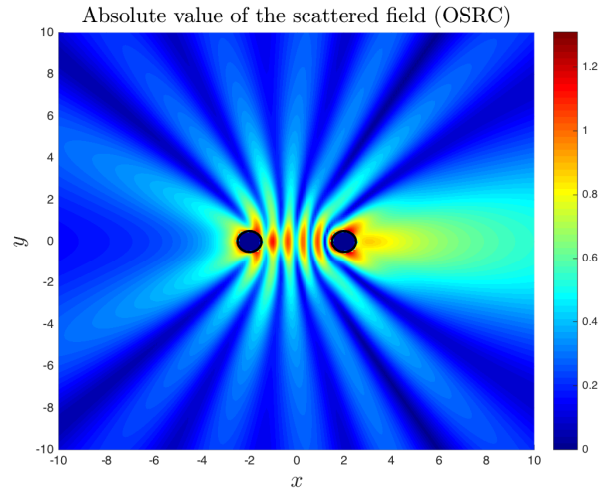

(b) $\operatorname{OSRC}^{N_{r}, \theta_{r}, 2}$ solution

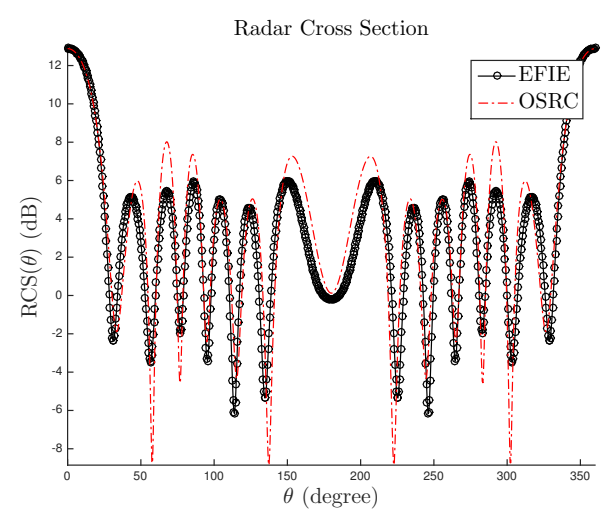

(c) RCS comparison

Figure 6: Scattering by two circular cylinders: comparison of the amplitude of the scattered fields for (a): the reference solution and (b): $\left.\operatorname{OSRC}^{N_{r}, \theta_{r}, 2}\right)$. The RCS are reported on Figure (c). The chosen parameters are: $a_{1}=a_{2}=a=0.5$, and $b=4$. The wavenumber $k$ is such that $k a=2.5$ and the angle of incidence is $\alpha=0$. 


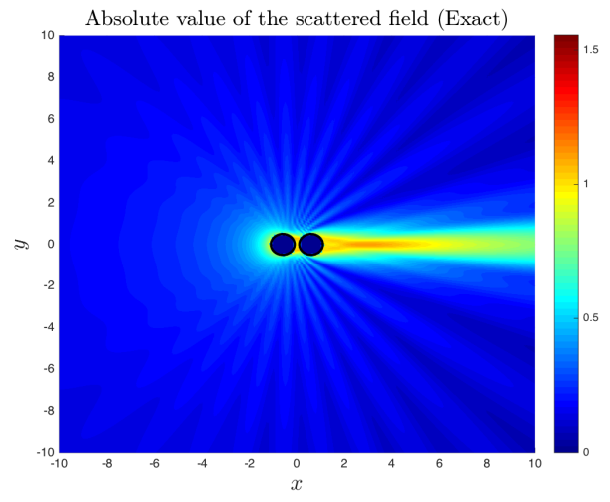

(a) Reference solution

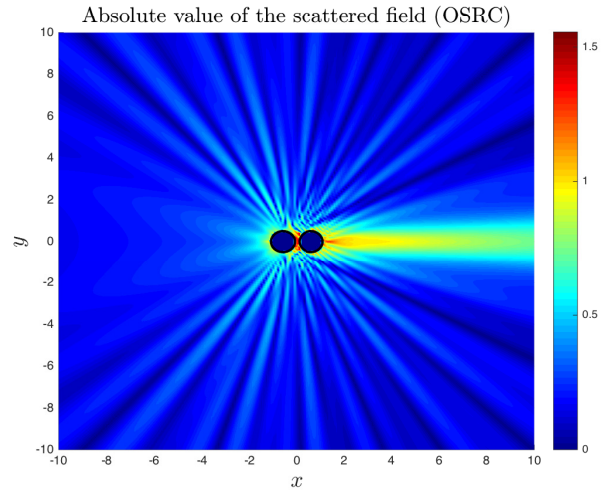

(b) $\operatorname{OSRC}^{N_{r}, \theta_{r}, 2}$ solution

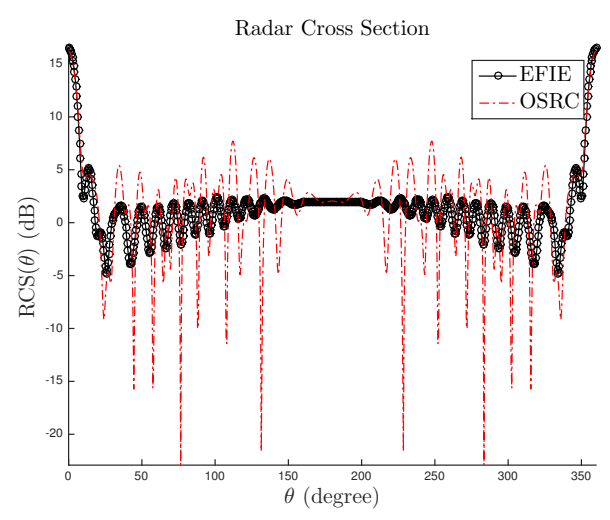

(c) RCS comparison

Figure 7: Scattering by two circular cylinders: comparison of the amplitude of the scattered fields for (a): the reference solution and (b): $\left.\operatorname{OSRC}^{N_{r}, \theta_{r}, 2}\right)$. The RCS are reported on Figure (c). The chosen parameters are: $a_{1}=a_{2}=a=0.5$, and $b=1.2$. The wavenumber $k$ is such that $k a=15$ and the angle of incidence is $\alpha=0$. 


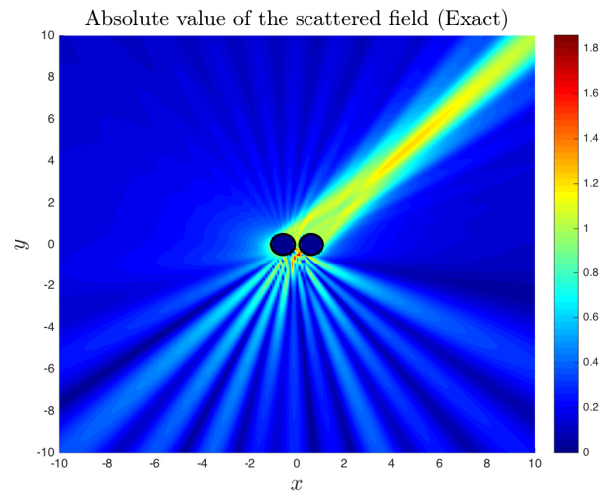

(a) Reference solution

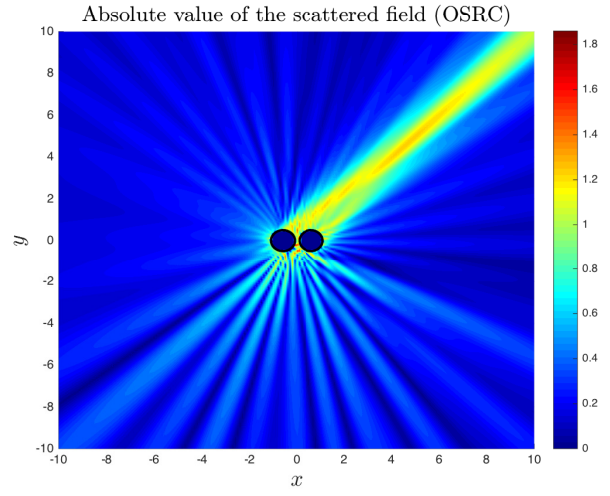

(b) $\operatorname{OSRC}^{N_{r}, \theta_{r}, 2}$ solution

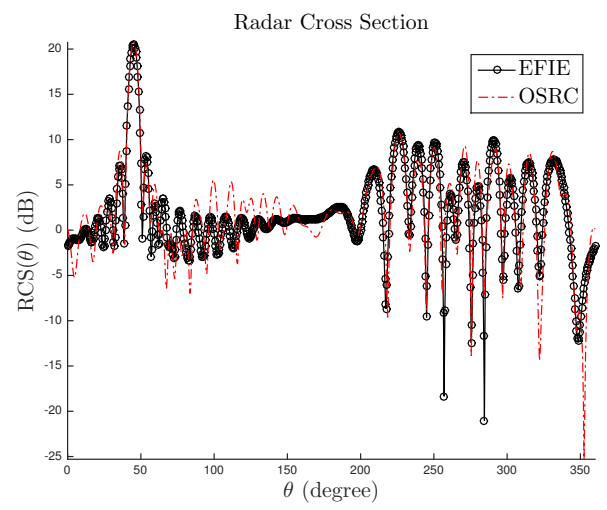

(c) RCS comparison

Figure 8: Scattering by two circular cylinders: comparison of the amplitude of the scattered fields for (a): the reference solution and (b): $\operatorname{OSRC}^{N_{r}, \theta_{r}, 2}$ ). The RCS are reported on Figure (c). The chosen parameters are: $a_{1}=a_{2}=a=0.5$, and $b=1.2$. The wavenumber $k$ is such that $k a=15$ and the angle of incidence is $\alpha=\pi / 4$. 


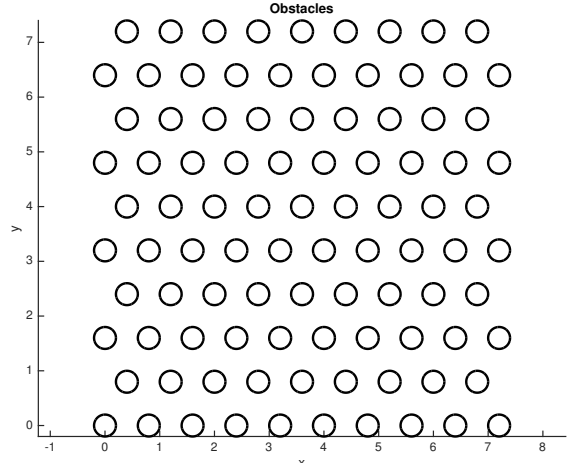

(a) Triangular lattice

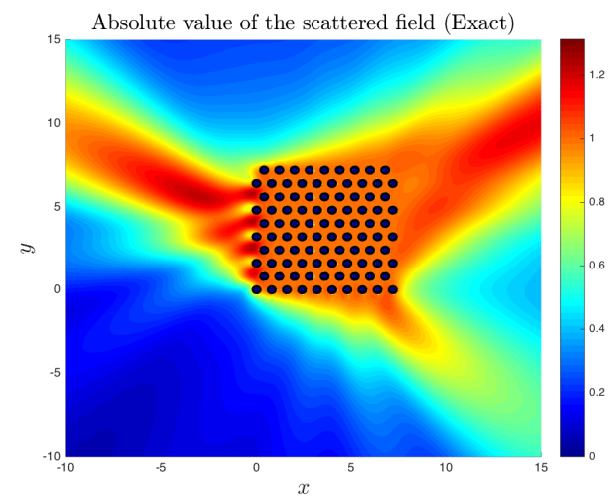

(c) Reference solution

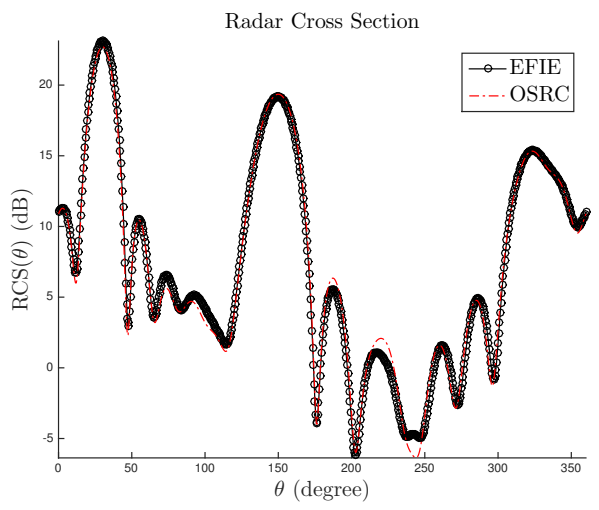

(b) RCS comparison

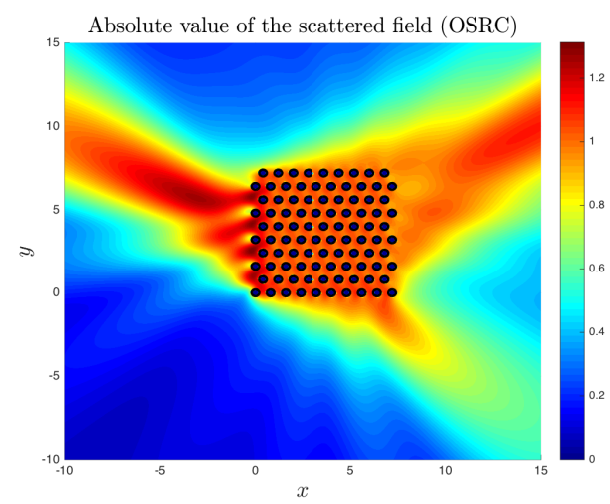

(d) $\operatorname{OSRC}^{N_{r}, \theta_{r}, 2}$ solution

Figure 9: Scattering by a triangular lattice $\left(M_{x}=10\right.$ and $M_{y}=10$ resulting in 95 circular scatterers (Figure (a)). The RCS are reported on Figure (b) and the comparison of the amplitude of the scattered fields is given on Figure (c) for the reference solution and (d) for the $\mathrm{OSRC}^{N_{r}, \theta_{r}, 2}$ solution. The inter-center distance between the obstacles is $b_{x}=b_{y}=0.8$. The radii of the scatterers are fixed to $a=0.2$. The wave number is $k=2$ and the incidence angle is $\alpha=\pi / 6$. 


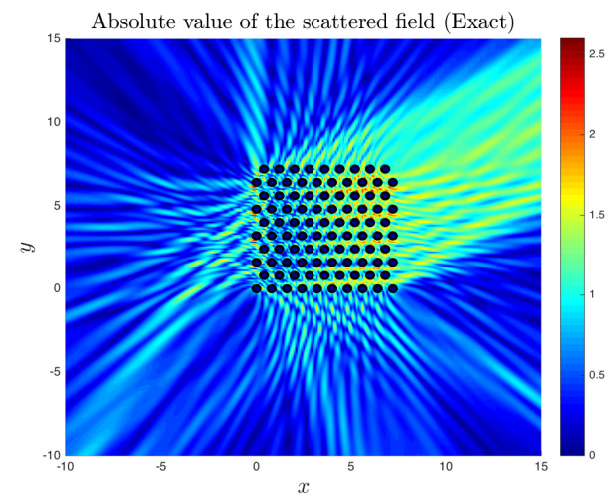

(a) Reference solution

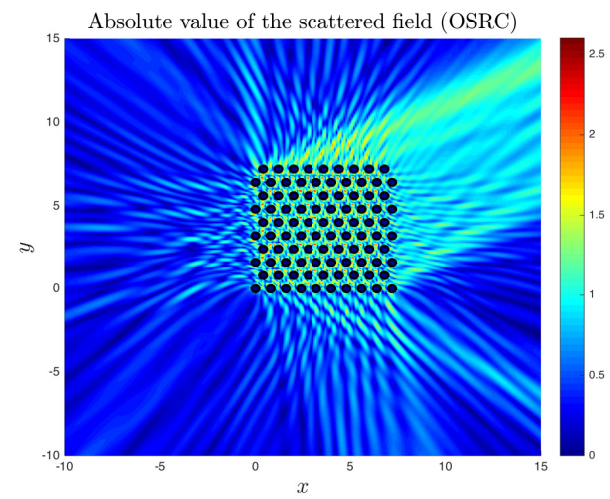

(b) $\operatorname{OSRC}^{N_{r}, \theta_{r}, 2}$ solution

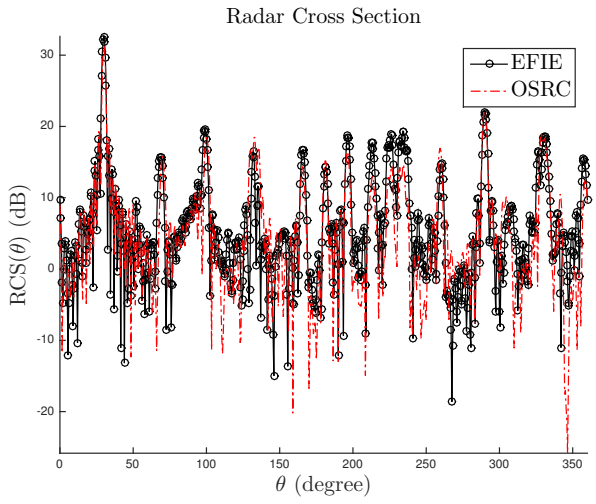

(c) RCS comparison

Figure 10: Scattering by a triangular lattice $\left(M_{x}=10\right.$ and $M_{y}=10$ resulting in 95 circular scatterers (Figure (a)). The RCS are reported on Figure (b) and the comparison of the amplitude of the scattered fields is given on Figure (c) for the reference solution and (d) for the $\mathrm{OSRC}^{N_{r}, \theta_{r}, 2}$ solution. The inter-center distance between the obstacles is $b_{x}=b_{y}=0.8$. The radii of the scatterers are fixed to $a=0.2$. The wave number is $k=15$ and the incidence angle is $\alpha=\pi / 6$. 


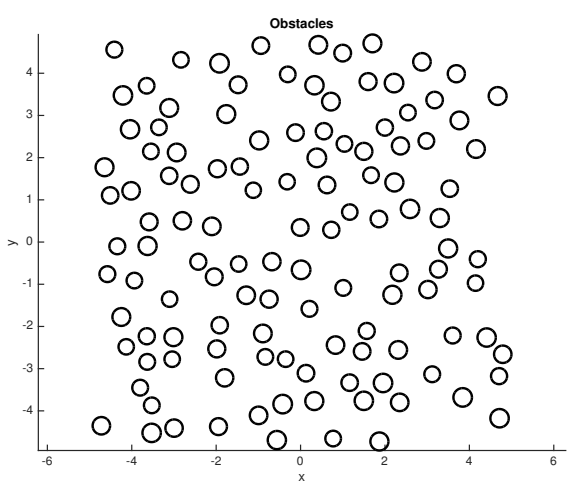

(a) Randomly distributed scatterers

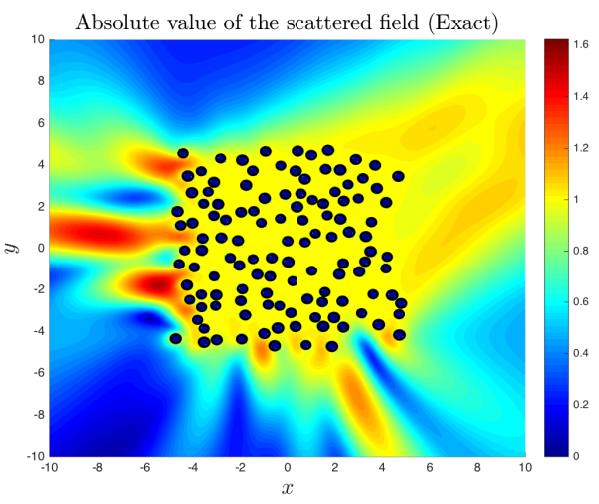

(c) Reference solution

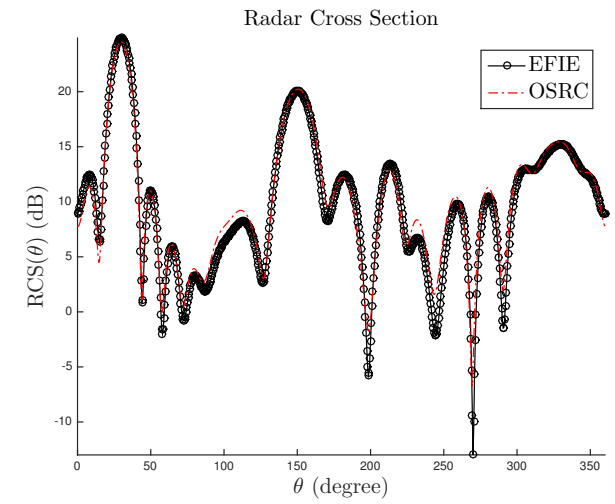

(b) RCS comparison

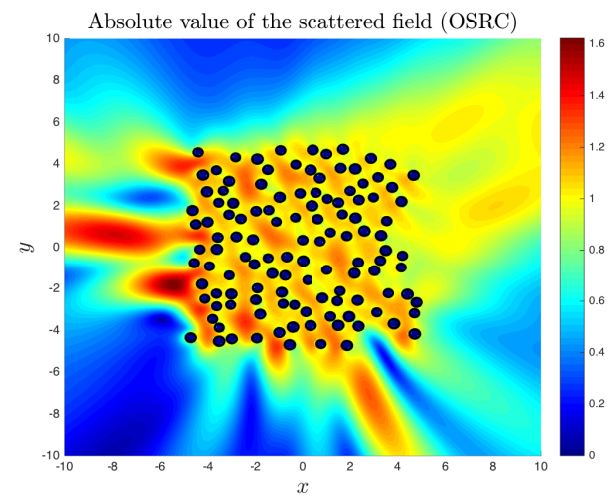

(d) $\operatorname{OSRC}^{N_{r}, \theta_{r}, 2}$ solution

Figure 11: Scattering by 120 randomly distributed scatterers with radii between 0.18 and 0.22 (see Figure (a)). The RCS are reported on Figure (b) and the comparison of the amplitude of the scattered fields is given on Figure (c) for the reference solution and (d) for the $\mathrm{OSRC}^{N_{r}, \theta_{r}, 2}$ solution. The wave number is $k=2$ and the incidence angle is $\alpha=\pi / 6$. 


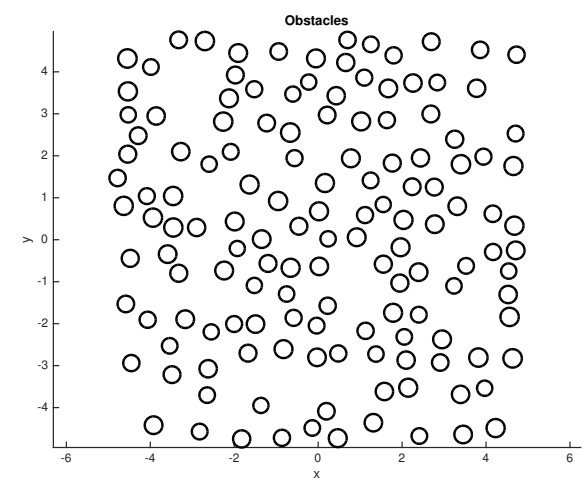

(a) Randomly distributed scatterers

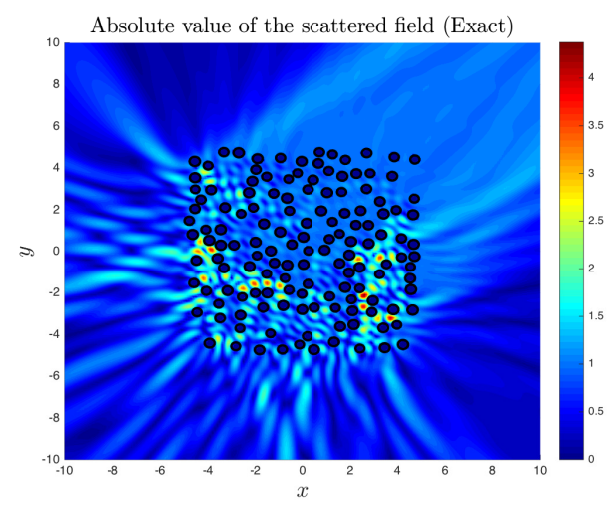

(c) Reference solution

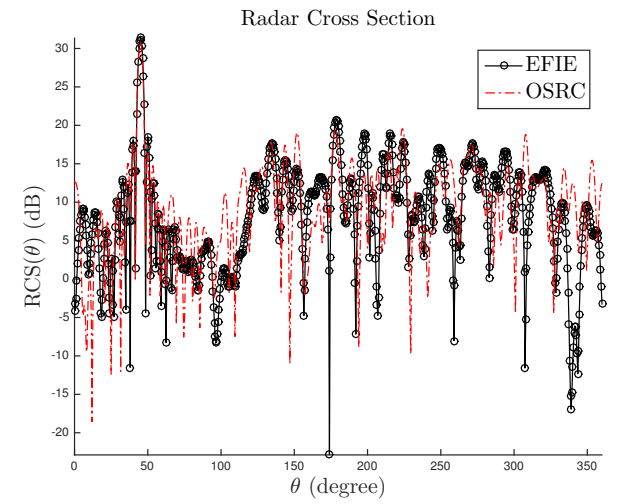

(b) RCS comparison

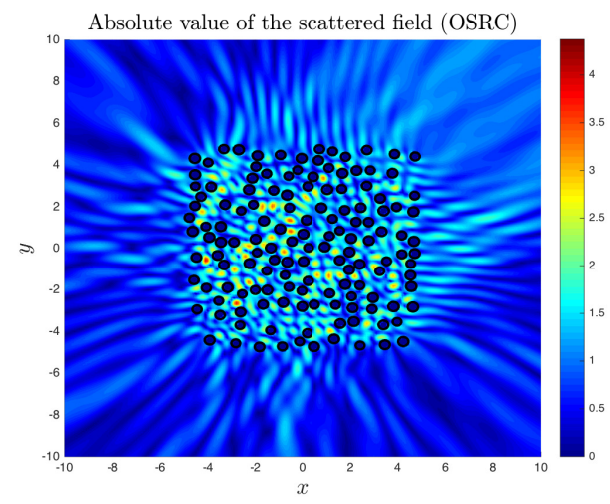

(d) $\operatorname{OSRC}^{N_{r}, \theta_{r}, 2}$ solution

Figure 12: Scattering by 100 randomly distributed scatterers with radii between 0.18 and 0.22 (see Figure (a)). The RCS are reported on Figure (b) and the comparison of the amplitude of the scattered fields is given on Figure (c) for the reference solution and (d) for the $\mathrm{OSRC}^{N_{r}, \theta_{r}, 2}$ solution. The wave number is $k=8$ and the incidence angle is $\alpha=\pi / 4$. 